\title{
Effect of Vine Load and Spraying Citric Acid on Fruiting of Superior Grapevines Grown Under Minia Region Conditions- Egypt
}

\author{
Ali, A.H. ${ }^{1}$; ${ }^{*}$ M.Kh. Uwakiem ${ }^{2}$ and H.M.M. Sayed ${ }^{1}$ \\ ${ }^{1}$ Hort. Dept. Fac. of Agric. Minia Univ. Egypt. \\ ${ }^{2}$ Viticulture Res. Dept. Hort. Res. Instit. ARC, Giza, Egypt. \\ *Email: maherkhaery@yahoo.com
}

Received on: 23/10/2016

Accepted for publication on: 2/11/2016

\begin{abstract}
During 2014 and 2015 seasons, Superior grapevines pruned to leave five vine loads namely $62,72,82,92$ and 102 eyes/vine and/ or spraying citric acid at 0.0 to $0.4 \%$ three times at growth start, just after berry setting and three weeks later.
\end{abstract}

The target was elucidating the effect of various vine loads and concentrations of citric acid on bud behavior, vine nutritional status, yield and berries quality.

Increasing vine loads from 62 to 102 eyes/vine caused a gradual promotion on fruiting buds $\%$, leaf area, pruning wood weight, cane thickness, chlorophylls a $\& \mathrm{~b}$, total chlorophylls, percentages of $\mathrm{N}, \mathrm{P}, \mathrm{K}$ and $\mathrm{Mg}$, berry setting $\%$, yield expressed in weight (kg.) and number of clusters/ vine, cluster compactness, cluster weight and dimensions, T.S.S.\%, T.S.S/acid and reducing sugars\% .Moreover, it caused a gradual reduction on bud burst $\%$, bud fertility $\%$, main shoot length, number of leaves/shoot, wood ripening coefficient, shot berries $\%$, berry weight and dimensions, juice $\%$ and titratable acidity $\%$. All the previous parameters except shot berries $\%$ and titratable acidity were gradually enhanced with the increasing concentrations of citric acid. Behavior of buds was unaffected by citric acid treatments.

The best results with regard to yield and berries quality of Superior grapevines grown under Minia region conditions were obtained by leaving 102 eyes per vine (on the basis of nine fruiting cane $X$ ten eyes plus six renewal spurs $X$ two eyes) during winter pruning plus spraying citric acid at $0.2 \%$ three times.

Keywords: Superior grapevines, vine loads, citric acid, behavior of buds, yield, quality of the berries.

\section{Introduction}

Nowadays, there is a widespread use of antioxidants. They are very effective in protecting plant cells from senescence and disorders (Robinson, 1973) as well as enhancing cell division, the biosynthesis of natural hormones such as IAA, GA3 and cy- tokinins, nutrients and water uptake, photosynthesis, biosynthesis of plant pigments and proteins as well as the biosynthesis of alpha ketoglutaric acid which is united with ammonia to form amino acids and proteins (Oretili, 1987; Samiullah et al., 1988; and Singh, et al., 2001). 
Pruning is the most important and vital cultural practice in the management of different grapevine cvs. It is a limiting factor for producing an economical yield and better quality of berries (Chapman, 1990). It is done to select the suitable vine load and maintain vine shape. It must be done each dormant season and it directly influences yield, fruit quality, vine vigour and hardiness. Proper pruning with the optimum vine load, spur length and proper date will result in improving yield quantitively and qualitatively without a reduction in hardiness during the following winter (El-Ashram, 1993 and Martin and Dunn, 2000). Improper pruning will have a detrimental effect on each of these important vine characteristics. In economic terms, the ability to prune correctly can make the differences between profit and loss. On average, grapevine will have 200 to 300 buds on mature cane capable of producing larger clusters. If the vines were allowed to produce shoots and fruits from these buds, the result would be a large crop that would reduce cane maturity, reduce the productivity of buds in the following season and decrease the size of the root system (Silvestroni et al., 1996; Valor and Bautista, 1995; Valachovic and Zembery, 2002; Tamura et al., 2002 and Ranpise et al., 2003). The main important problem that faces the grape production in most Egyptian vineyards is the determination of the suitable and productive bud load per vine. Bud load is the most important factor that affects yield and vine vigour (Omar and Abdel-Kawi, 2000). The lack of information about the suitable bud load per vine might be the main reason for the deterioration of vine growth in such region or at least for the low vine productivity. Low bud loaded vines produce an obvious stimulation on number of leaves which shade the clusters and impaired the fruit quality (Miller et al., 1993; Gao and Cahoon, 1994 and Samra, 1998).

El-Sese (2004) found that the number of bursting buds and bud fertility were increased gradually by increasing vine load from 38 to 84 buds /vine in Red Roomy grapevines.

Increasing cane length of Superior grapevines during winter pruning resulted in a gradual promotion on bud fertility coefficient and a progressive reduction on buds burst $\%$ (Rizk-Alla-Mervat and El-Zyat, 2005).

Hussein (2009); Fawzi et al., (2010); Abdel-Mohsen (2013) and Sourial et al., (2016) pointed out that there was a gradual reduction on bud burst $\%$ and promotion on vine nutritional status, yield and quality of the berries in different grapevine cvs. with increasing vine loads from 48 to 112 eyes.

Growth, vine nutritional status, yield and quality of Thompson seedless grapevines were clearly improved in response to treating the vines with a mixture of vitamins $E$, $\mathrm{K}, \mathrm{B}$ and $\mathrm{A}$ each at $25 \mathrm{ppm}$, amino acids at $250 \mathrm{ppm}$, citric acid at 1000 
ppm and folic acid at $25 \mathrm{ppm}$ over the control (Abdelaal and Aly., 2013).

Mohamed, et al., (2015) stated that all growth aspects, yield and berries quality of Superior grapevines were remarkably enhanced in response to treating the vines three times with vitamin $\mathrm{B}$ at $50 \mathrm{ppm}$ combined with ascorbic and citric acids each at $500 \mathrm{ppm}$.

The promoting effect of citric acid application on growth, vine nutritional status, yield and quality of the berries in different grapevine cvs. was supported by the results of Abd El- Galil , (2015); Ebrahiem, (2015) and Mohamed-Attiat, (2016).

The main target of this study was examining the effect of different vine loads and foliar application of citric acid on behavior of buds, vegetative growth characteristics, vine nutritional status, yield and quality of berries of Superior grapevines grown under Minia region conditions.

\section{Material and Methods}

This study was carried out during 2014 and 2015 seasons on 150 uniform in vigour own-rooted 13years old Superior grapevines grown in a private vineyard located at Abwan village, Matay district, Minia Governorate where the texture of the soil is clay, well drained and with a water table not less than two meters deep. All the selected vines are planted at $2.5 \times 3.0 \mathrm{~m}$ apart. The chosen vines (150 vines) were pruned during the first week of January in the two seasons using cane pruning method with the assistance of Gable supporting system. Vine load was differed according to the present treatments. Surface irrigation system was followed using Nile water containing 150 ppm salinity.

Mechanical, physical and chemical analysis of the tested soil were carried out at the start of the experiment according to the procedures of Wilde et al., (1985) and the data are shown in Table (1).

Table 1. Analysis of the tested soil:

\begin{tabular}{|l|c|}
\hline \multicolumn{1}{|c|}{ Constituents } & Values \\
\hline Particle size distribution: & \\
\hline Sand \% & 11.0 \\
\hline Silt $\%$ & 20.5 \\
\hline Clay $\%$ & 68.5 \\
\hline Texture & Clay \\
\hline $\mathrm{pH}(1: 2.5$ extract) & 8.05 \\
\hline $\mathrm{EC}(1: 2.5$ extract $)\left(\mathrm{dsm}^{-1}\right) 1 \mathrm{~cm} / 25^{\circ} \mathrm{C}$. & 1.03 \\
\hline O.M. $\%$ & 1.88 \\
\hline $\mathrm{CaCO}_{3} \%$ & 2.55 \\
\hline Total $\%$ & 0.10 \\
\hline Available $\mathrm{P}($ Olsen, $\mathrm{ppm})$ & 4.22 \\
\hline Available $\mathrm{K}$ (ammonium acetate, $\mathrm{ppm})$ & 400 \\
\hline
\end{tabular}


Except those dealing with the present treatments (pruning and application of citric acid via foliage), all the selected vines (150 vines) received the usual horticultural practices which are commonly used in the vineyard.

This study included twenty-five treatments from two factors (A\&B). The first factor (A) contained the following five levels of vine loads:-

a1) Leaving 62 eyes/vine (on the basis of five fruiting canes X 10 eyes plus six renewal spurs $X 2$ eyes).

a2) Leaving 72 eyes/vine (on the basis of six fruiting canes $\mathrm{X} 10$ eyes plus six renewal spurs $\mathrm{X} 2$ eyes).

a3) Leaving 82 eyes/vine (on the basis of seven fruiting canes X 10 eyes plus six renewal spurs $\mathrm{X} 2$ eyes). a4) Leaving 92 eyes/vine (on the basis of eight fruiting canes $\mathrm{X} 10$ eyes plus six renewal spurs $X 2$ eyes).

a5) Leaving 102 eyes/vine (on the basis of nine fruiting canes $\mathrm{X} 10$ eyes plus six renewal spurs $X 2$ eyes).

The second factor (B) included the following five concentration of citric acid: b1) $0.0 \%$, b2) $0.05 \%$, b3) $0.1 \%$, b4) $0.2 \%$ and b5) $0.4 \%$. Each treatment was replicated three times, two vines per each.

Winter pruning leaving the previous five vine loads was carried out at the first week of January. during 2014 and 2015 seasons. Citric acid was sprayed three times at growth start (last week of February.), just after berry setting stage (mid. of April.) and at three weeks later (1st week of May).
Triton B as a wetting agent was added to all citric acid solutions at $0.05 \%$. Spraying was established till runoff (2L/vine). Control treatment was sprayed with water containing Triton B.

Randomized complete block design (RCBD) in split plot arrangement was followed. The first factor i.e. five vine loads occupied the main plots and the second one namely five concentrations of citric acid ranked the subplots. The experiment consisted of twenty-five treatments, each treatment was replicated three times and two vines per each.

During both seasons, the following parameters were recorded:

1- Behavior of buds including percentages of bud burst, fruiting buds and bud fertility.

2- Vegetative growth characteristics namely main shoot length $(\mathrm{cm})$, number of leaves/shoot, leaf area (Ahmed and Morsy, 1999); wood ripening coefficient (Bouard, 1966); pruning wood weight per vine (kg.) and cane thickness $(\mathrm{cm})$.

3- Chlorophylls (a \& b), total chlorophylls (mg/100g F.W) (vonWettstein, 1957).

4- Percentages of $\mathrm{N}, \mathrm{P}, \mathrm{K}$ and $\mathrm{Mg}$ on dry weight basis (Wild et al., 1985 and Balo et al., 1988).

5- Percentage of berry setting, yield expressed as weight $(\mathrm{kg})$ and number of clusters of vine as well as compactness (number of berries/cluster length), weight, length and shoulders $(\mathrm{cm})$ of cluster.

6- Percentage of shot berries. 
7- Physical and chemical characteristics of the berries namely berry weight and dimensions (longitudinal and equatorial, in $\mathrm{cm}$ ), juice $\%$, T.S.S.\%, titratable acidity as tartaric $\mathrm{acid} / 100 \mathrm{ml}$ juice (A.O.A.C, 2000) and reducing sugars\% (Lane and Eynon, 1965 and A.O.A.C, 2000).

Statistical analysis was done using Randomized complete block design (RCBD) (according to Mead et al., 1993). New L.S.D at 5\% parameter was used for all comparisons among various treatment means.

\section{Results and Discussion}

\section{1- Behavior of buds:}

It is clear from the obtained data in Table (2) that increasing vine loads from 62 to 102 eyes/vines caused a significant and gradual promotion on the percentage of fruiting buds and at the same time caused a progressive reduction on both the percentages of bud burst and bud fertility.

Citric acid treatments had no significant effect on the percentages of bud burst, fruiting buds and bud fertility.

The maximum values of buds burst $\%$ and bud fertility were recorded on the vines that pruned to leave 62 eyes/vine regardless the application of citric acid. Leaving 102 eyes/vine gave the maximum percentage of fruiting buds. These results were true during both seasons.

The reduction of the percentage of bud burst at the higher vine loads might be attributed to the vine nutritional status and the balance between inorganic and organic foods (Weaver, 1976).

These results regarding the effect of vine load on behavior of buds are in harmony with these obtained by Abdalla, (2003); El-Sese, (2004) and Hussein, (2009).

\section{Vegetative growth character- istics:}

It is obvious from the data in Tables ( $2 \& 3 \& 4)$ that the varying number of eyes left per vine during winter pruning lead to significant differences on the main shoot length, number of leaves/shoot, wood ripening coefficient, leaf area, pruning wood weight and cane thickness. Increasing vine loads from 62 to 102 eyes/vine caused a progressive promotion on the leaf area, pruning wood weight and cane thickness and a reduction on main shoot length, number of leaves/shoot and wood ripening coefficient. Treating the vines three times with citric acid at 0.05 to $0.4 \%$ caused significant promotion on all the aforementioned growth characteristics over the control. Using the higher concentrations of citric acid namely $0.4 \%$ achieved the highest values of growth traits.

Spraying citric acid at $0.4 \%$ besides leaving 102 eyes/vine during winter pruning gave the maximum leaf area, pruning wood weight, cane thickness and the highest values of main shoot length, number of leaves/shoot and wood ripening coefficient were recorded on the vines that were pruned to leave 62 eyes/vine and received three sprays of 
citric acid at $0.4 \%$. These results were true during both seasons.

The promotion on the leaf area, pruning wood weight and cane thickness at the higher levels of vine loads might be attributed to the reduction on the percentage of bud burst and the lowest shoot length and number of leaves per shoot. The reduction on shoot length was surely reflected on enhancing cane thickness (Weaver, 1976). The promotion effect of citric acid on growth weight could be attributed to its effect in enhancing cell division, the biosynthesis of organic foods and the tolerance of plants to biotic and abiotic stresses (Oretili, (1987); Samiullah et al., (1988) and Singh et al., (2001)).

The results regarding the effect of vine load on growth aspects are in agreement with those obtained by Abd El-Mohsen, (2013) and Sourial et al., (2016).

The stimulating effects of citric acid on growth traits support the results of Abdel-Aal and Aly (2013) and Ebrahiem, (2015).

\section{2- Leaf chemical composition:}

Data in Tables (4\&5) clearly show that increasing vine loads from 62 to 102 eye/vine and concentrations of citric acid from 0.0 to $0.4 \%$ caused a gradual and significant promotion on chlorophylls (a \& b), total chlorophylls and percentages of N, P, K and $\mathrm{Mg}$ in the leaves.

The maximum values of chlorophylls (a \& b), total chlorophylls, N, $\mathrm{P}, \mathrm{K}$ and $\mathrm{Mg}$ in the leaves were recorded on the vines pruned to leave 102 eyes/vine and sprayed three times with citric acid at $0.4 \%$. These findings were true during both seasons.

The increase on the leaf chemical constituents at the higher levels of vine load might be attributed to the promotion on the leaf area which resulted in enhancing photosynthesis process as well as the reduction on main shoot length and number of leaves which aids in reducing the depletions of these nutrients (Weaver, 1976).

The promotion effect of citric acid on leaf pigments and different nutrients might be attributed to its positive action on enhancing the biosynthesis of sugars and plant pigments as well as the uptake of nutrients especially $\mathrm{N}$ and $\mathrm{Mg}$ (Singh et al., 2001).

The results of vine load effcets are in concordance with those obtained by Abdalla, (2003); El-Sese, (2004); Hussein, (2009) and Fawzi et al., (2010).

These results regarding the stimulating effect of citric acid on leaf pigments, $\mathrm{N}, \mathrm{P}, \mathrm{K}$ and $\mathrm{Mg}$ are in harmony with those obtained by Mohamed et al., (2015) and MohamedAttiat, (2016).

\section{3- Berry setting, yield and cluster characteristics:}

It is evident from data in Tables $(6 \quad \& \quad 7)$ that increasing vine loads frpm 62 to 102 eyes/vine caused a gradual and significant promotion on the percentage of berry setting, yield expressed in weight and number of clusters/vine as well as compactness, weight, length and shoulder of cluster. 
Spraying citric acid at 0.05 to $0.4 \%$ significantly improved the percentage of berry setting, yield and number of clusters/vine as well as compactness, weight, length and shoulder of cluster over the check treatment. The promotion was proportional to the increase in concentrations. Increasing concentrations of citric acid from 0.2 to $0.4 \%$ failed to show significant promotion on these parameters. Therefore, from economical point of view, it is recommended to spraying citric acid at $0.2 \%$.

Pruning Superior grapevines during winter to leave 102 eyes/vine beside spraying citric acid at $0.2 \%$ gave an economical yield. Under such promised treatment, yield per vine reached 8.5 and $12.5 \mathrm{~kg}$ during both seasons, respectively. Leaving 62 eyes/vine without using citric acid gave the lowest values. Pruning and citric acid treatments had no significant effect on number of clusters/vine in the first season of study. These results were true during both seasons.

The promoting effect of proper vine load (102 eyes/vine) as well as application of citric acid on growth, vine nutritional status, berry setting and cluster weight could result in improving the yield.

These results regarding the positive effect of adjusting vine load on berry setting, yield and cluster characteristics are in the same line with those obtained by El-Sese, (2004); Hussein, (2009); Fawzi et al., (2010) and Sourial et al., (2016).
The promotion effect of citric acid application on berry setting, yield and cluster characteristics supports the results of Mohamed et al., (2015); Ebrahiem, (2015) and Abd ElGalil, (2015) on different grapevine cvs.

\section{4- Percentage of shot berries:}

It is clear from the data in Table (7) that increasing vine loads from 62 to 102 eyes/vine caused a significant and gradual increase on the percentage of shot berries. The highest values were recorded on the clusters harvested from vines pruned to leave 102 eyes/vine. The lowest values were recorded on the clusters harvested from vines pruned to leave 62 eyes/vine.

Treating the vines with citric acid at 0.05 to $0.4 \%$ significantly reduced the percentage of shot berries over the check treatment. The reduction was proportional to the increase in concentrations of citric acid. No significant reduction was observed among the higher two concentrations of citric acid namely 0.2 and $0.4 \%$.

The lowest values of shot berries $\%$ were recorded on the vines that pruned to 62 eyes/vine plus spraying citric acid at $0.4 \%$ during both seasons.

The higher values of shot berries under higher vine loads might be attributed to the great depletion of various nutrients in forming more clusters which result in imbalance between various nutrients (Weaver, 1976).

The beneficial effect of citric acid in enhancing the uptake of most 
nutrients and water could explain the present results (Singh et al, 2001).

The investigated effect of vine loads on shot berries was emphasized by the results of Sourial et al., (2016).

The results of Abd El- Galil, (2015) and Ebrahiem, (2015) emphasized the effect of citric acid on reducing shot berries $\%$.

\section{5- Berries quality:}

Data in Table (8\&9) clearly showed that the increasing vine loads from 62 to 102 eyes/vine caused a gradual reduction on berry weight and dimensions (longitudinal and equatorial), juice $\%$, and total acidity $\%$ and a progressive promotion on T.S.S. \%, T.S.S./acid and reducing sugars.

Treating the vines with citric acid at 0.05 to $0.4 \%$ resulted in a significant promotion on physical and chemical characteristics of the berries in terms of increasing berry weight and dimensions, juice $\%$, T.S.S\%, T.S.S/acid, reducing sugars $\%$ and decreasing titratable acidity $\%$ over the check treatment. There was a gradual promotion on fruit quality with increasing concentrations of citric acid. Meaningless promotion on fruit quality was observed among the higher two concentrations namely 0.2 and $0.4 \%$.

The best results with regard to quality of the berries were obtained with carrying out pruning to leave
102 eyes/vine and foliar application of citric acid at $0.2 \%$. These results were true during both seasons.

The previous positive action of the proper pruning (102 eyes/vine) on the leaf area, leaf pigments and $\mathrm{Mg}$ was surely reflected on advancing maturity and enhancing fruit quality.

The beneficial effects of citric acid on fruit quality might be attributed to its effect in enhancing the biosynthesis of sugars and plant pigments as well as the uptake of $\mathrm{Mg}$ that resulted in improving and advancing fruit maturity and fruit quality (Oretili, (1987) and Singh et al., (2001).

The present effect of the proper pruning on quality of the berries are in accordance with the results of Hussein, (2009) and Sourial et al., (2016).

The results of Abd El-Galil, (2015) and Ebrahiem, (2015) emphasized the present results regarding the promoting effect of citric acid on fruit quality.

\section{Conclusion}

The best results with regard to yield and fruit quality of Superior grapevine grown under Minia region conditions were obtained by leaving 102 eyes (on the basis of nine fruiting cane $\mathrm{X}$ ten eyes plus six renewal spur $\mathrm{X}$ two eyes during winter pruning plus spraying citric acid at $0.2 \%$ three times. 
Table 2. Effect of different vine loads and citric acid concentrations on the percentages of bud burst, fruiting buds, bud fertility and main shoot length $(\mathrm{cm})$ of Superior grapevines during 2014 and 2015 seasons.

\begin{tabular}{|c|c|c|c|c|c|c|c|c|c|c|c|c|c|c|c|c|c|c|c|c|c|c|c|c|}
\hline \multirow{4}{*}{ Vine laod levels (A) } & \multicolumn{12}{|c|}{ Bud burst \% } & \multicolumn{12}{|c|}{ Fruiting buds \% } \\
\hline & \multicolumn{6}{|c|}{2014} & \multicolumn{6}{|c|}{2015} & \multicolumn{6}{|c|}{2014} & \multicolumn{6}{|c|}{2015} \\
\hline & \multicolumn{24}{|c|}{ Citric acid concentrations (B) } \\
\hline & $\begin{array}{l}b 1 \\
0.0\end{array}$ & \begin{tabular}{c|} 
b2 \\
0.05
\end{tabular} & b3 0.1 & b4 0.2 & b5 0.4 & Mean A & $\begin{array}{l}\text { b1 } \\
0.0\end{array}$ & \begin{tabular}{|c|c} 
b2 \\
0.05 \\
\end{tabular} & $\begin{array}{l}b 3 \\
0.1\end{array} \mid$ b & b4 0.2 & b5 0.4 & Mean A & $\begin{array}{l}\text { b1 } \\
0.0\end{array}$ & \begin{tabular}{|c|}
$\mathbf{b 2}$ \\
0.05
\end{tabular} & b3 0.1 & b4 0.2 & b5 0.4 & Mean A & $\begin{array}{l}\text { b1 } \\
0.0\end{array}$ & \begin{tabular}{|c|} 
b2 \\
0.05 \\
\end{tabular} & b3 0.1 & b4 0.2 & b5 0.4 & Mean A \\
\hline a1 62eyes $(5 \times 10+6 \times 2)$ & 75.0 & 75.1 & 75.1 & 75.1 & 75.1 & 75.1 & 76.1 & \begin{tabular}{|l|l|l}
76.2 & 7 \\
\end{tabular} & 76.3 & 76.5 & 76.5 & 76.3 & 41.9 & 42.0 & 42.0 & 42.0 & 42.0 & 42.0 & 43.1 & 43.2 & 43.3 & 43.3 & 43.3 & 43.2 \\
\hline a2 72 eyes $(6 \times 10+6 \times 2)$ & 74.0 & 74.1 & 74.1 & 74.1 & 74.1 & 74.1 & 75.0 & 75.17 & 75.3 & 75.4 & 75.4 & 75.2 & 43.1 & 43.3 & 43.3 & 43.3 & 43.3 & 43.3 & 44.5 & 44.6 & 44.7 & 44.8 & 44.8 & 44.7 \\
\hline a3 82 eyes $(7 \times 10+6 \times 2)$ & 72.0 & 73.0 & 73.1 & 73.1 & 73.2 & 73.1 & 74.1 & \begin{tabular}{|l|l|l|l}
74.2 & 7 \\
\end{tabular} & 74.2 & 74.3 & 74.3 & 74.2 & 44.3 & 44.7 & 44.7 & 44.7 & 44.8 & 44.6 & 46.9 & 46.0 & 46.0 & 46.0 & 46.0 & 46.2 \\
\hline a4 92eyes $(8 \times 10+6 \times 2)$ & 72.0 & 72.2 & 72.2 & 72.2 & 72.2 & 72.2 & 73.3 & \begin{tabular}{|l|l}
73.5 & 7 \\
\end{tabular} & 73.5 & 73.5 & 73.5 & 73.5 & 46.0 & 46.1 & 46.1 & 46.1 & 46.1 & 46.1 & 48.0 & 48.0 & 48.0 & 48.0 & 48.0 & 48.0 \\
\hline a5 102eyes $(8 \times 10+6 \times 2)$ & 71.0 & 71.3 & 71.3 & 71.3 & 71.3 & 71.3 & 72.4 & \begin{tabular}{|c|c|c|}
72.5 & 7 \\
\end{tabular} & 72.5 & 72.5 & 72.5 & 72.5 & 47.8 & 48.0 & 48.0 & 48.0 & 48.0 & 48.0 & 49.3 & 49.5 & 49.5 & 49.5 & 49.6 & 49.5 \\
\hline Mean (B) & 73.0 & \begin{tabular}{|l|l}
73.1 \\
\end{tabular} & 73.2 & 73.2 & 73.2 & & 74.2 & \begin{tabular}{|l|l|l|l|}
74.3 & 7 \\
\end{tabular} & 74.4 & 74.4 & 74.4 & & 44.6 & 44.8 & 44.8 & 44.8 & 44.9 & & 46.4 & 46.3 & 46.3 & 46.3 & 46.3 & \\
\hline New L.S.D at 5\% & & & $\begin{array}{l}\text { A } \\
0.8\end{array}$ & $\begin{array}{l}\text { B } \\
\text { NS }\end{array}$ & $\begin{array}{c}\text { AB } \\
\text { NS }\end{array}$ & & & & $\begin{array}{l}\text { A } \\
0.8\end{array}$ & $\begin{array}{l}\text { B } \\
\text { NS }\end{array}$ & $\begin{array}{c}\text { AB } \\
\text { NS }\end{array}$ & & & & $\begin{array}{l}\text { A } \\
0.9\end{array}$ & $\begin{array}{l}\text { B } \\
\text { NS }\end{array}$ & $\begin{array}{c}\text { AB } \\
\text { NS }\end{array}$ & & & & $\begin{array}{l}A \\
1.0\end{array}$ & $\begin{array}{l}\text { B } \\
\text { NS }\end{array}$ & $\begin{array}{c}\text { AB } \\
\text { NS }\end{array}$ & \\
\hline \multirow{3}{*}{ Character } & \multicolumn{12}{|c|}{ Bud fertility \% } & \multicolumn{12}{|c|}{ Main shoot length (cm) } \\
\hline & \multicolumn{6}{|c|}{2014} & \multicolumn{6}{|c|}{2015} & \multicolumn{6}{|c|}{2014} & \multicolumn{6}{|c|}{2015} \\
\hline & \begin{tabular}{|l|}
$\mathrm{b} 1$ \\
0.0 \\
\end{tabular} & \begin{tabular}{|c|} 
b2 \\
0.05 \\
\end{tabular} & b3 0.1 & b40.2 & b5 0.4 & Mean A & \begin{tabular}{l|l}
$\mathrm{b1}$ \\
0.0 \\
0.0
\end{tabular} & \begin{tabular}{|l|l|}
$b 2$ & \\
0.05 & \\
\end{tabular} & \begin{tabular}{|l|}
$\mathrm{b} 3$ \\
0.1 \\
\end{tabular} & $\mathrm{~b} 40.2$ & b5 0.4 & Mean $A$ & \begin{tabular}{l|l|}
$b 1$ \\
0.0 \\
0
\end{tabular} & \begin{tabular}{|c|} 
b2 \\
0.05 \\
\end{tabular} & b3 0.1 & $\mathrm{~b} 40.2$ & b5 0.4 & Mean A & $\begin{array}{l}\mathrm{b1} \\
0.0 \\
\end{array}$ & $\begin{array}{l}\mathrm{b2} \\
0.05 \\
0.0\end{array}$ & b3 0.1 & $\mathrm{~b} 40.2$ & b5 0.4 & Mean A \\
\hline a1 62eyes $(5 \times 10+6 \times 2)$ & 53.3 & 56.0 & 56.0 & 56.0 & 56.0 & 55.5 & 55.1 & \begin{tabular}{l|l}
60.4 & 6 \\
\end{tabular} & \begin{tabular}{l|l|}
65.4 \\
\end{tabular} & 65.4 & 70.5 & 63.4 & 91.0 & 100.0 & 103.0 & 105.0 & 105.5 & 102.5 & 100.0 & 102.0 & 104.0 & 106.0 & 106.0 & 103.6 \\
\hline a2 72 eyes $(6 \times 10+6 \times 2)$ & 47.3 & 47.3 & 47.3 & 47.3 & 47.3 & 47.3 & 51.15 & \begin{tabular}{l|l}
55.4 & 6 \\
\end{tabular} & \begin{tabular}{l|l|}
61.9 \\
\end{tabular} & 64.0 & 68.6 & 60.2 & 97.0 & 98.0 & 101.0 & 103.0 & 103.3 & 100.5 & 96.0 & 97.0 & 99.0 & 99.9 & 100.0 & 98.4 \\
\hline a3 82eyes $(7 \times 10+6 \times 2)$ & 41.2 & 41.1 & 41.0 & 41.0 & 41.0 & 41.1 & 50.15 & \begin{tabular}{|l|l}
53.8 & 5 \\
\end{tabular} & \begin{tabular}{|l|l|}
59.9 \\
\end{tabular} & 60.8 & 60.8 & 56.3 & 95.0 & 96.5 & 97.9 & 99.0 & 99.3 & 97.6 & 94.0 & 96.0 & 98.0 & 100.0 & 100.0 & 97.6 \\
\hline a4 92eyes $(8 \times 10+6 \times 2)$ & 36.5 & 36.3 & 36.3 & 36.3 & 36.3 & 36.3 & \begin{tabular}{l|l}
46.1 & 2 \\
\end{tabular} & \begin{tabular}{l|l}
49.3 & 5 \\
\end{tabular} & 51.0 & 54.4 & 54.4 & 51.0 & \begin{tabular}{l|l|}
91.0 \\
\end{tabular} & 92.3 & 94.0 & 95.0 & 95.1 & 93.5 & \begin{tabular}{|l|}
92.0 \\
\end{tabular} & \begin{tabular}{|l|}
94.0 \\
\end{tabular} & 96.0 & 98.0 & 98.0 & 95.6 \\
\hline a5 102eyes $(8 \times 10+6 \times 2)$ & 32.8 & 32.7 & 32.7 & 34.3 & 34.2 & 33.3 & \begin{tabular}{|l|l}
44.5 \\
\end{tabular} & \begin{tabular}{|l|l|}
45.9 & 4 \\
\end{tabular} & $\begin{array}{l}47.5 \\
\end{array}$ & 49.0 & 49.0 & 47.2 & \begin{tabular}{|l|}
88.0 \\
8
\end{tabular} & 89.0 & 90.9 & 92.0 & 92.1 & 90.4 & 90.0 & 92.0 & 94.0 & 96.0 & 96.3 & 93.7 \\
\hline Mean (B) & 42.2 & 42.7 & 42.7 & 43.0 & 43.0 & & $49.4:$ & \begin{tabular}{|l|l}
53.0 & 5 \\
\end{tabular} & 56.3 & 58.7 & 60.7 & & 94.0 & 99.2 & 97.4 & 98.8 & 99.1 & & 94.4 & 96.2 & 98.2 & 100.0 & 100.0 & \\
\hline New L.S.D at 5\% & & & $\begin{array}{l}A \\
1.0\end{array}$ & $\begin{array}{l}\text { B } \\
\text { NS }\end{array}$ & $\begin{array}{c}\text { AB } \\
\text { NS }\end{array}$ & & & & $\begin{array}{l}\text { A } \\
0.0\end{array}$ & $\begin{array}{l}\text { B } \\
0.9\end{array}$ & $\begin{array}{c}\text { AB } \\
2.0\end{array}$ & & & & $\begin{array}{l}\text { A } \\
0.9\end{array}$ & $\begin{array}{l}\text { B } \\
1.0\end{array}$ & $\begin{array}{c}\mathrm{AB} \\
2.2\end{array}$ & & & & $\begin{array}{l}\text { A } \\
1.0\end{array}$ & $\begin{array}{l}B \\
1.0\end{array}$ & $\begin{array}{c}\text { AB } \\
2.4\end{array}$ & \\
\hline
\end{tabular}


Table 3. Effect of different vine loads and citric acid concentrations on some vegetative growth characteristics of Superior grapevines during 2014 and 2015 seasons.

\begin{tabular}{|c|c|c|c|c|c|c|c|c|c|c|c|c|c|c|c|c|c|c|c|c|c|c|c|c|}
\hline \multirow{4}{*}{$\begin{array}{l}\text { Vine laod levels } \\
\text { (A) }\end{array}$} & \multicolumn{12}{|c|}{ No. of leaves /shoot } & \multicolumn{12}{|c|}{ Leaf area $(\mathrm{cm})^{2}$} \\
\hline & \multicolumn{6}{|c|}{2014} & \multicolumn{6}{|c|}{2015} & \multicolumn{6}{|c|}{2014} & \multicolumn{6}{|c|}{2015} \\
\hline & \multicolumn{24}{|c|}{ Citric acid concentrations (B) } \\
\hline & b10.0 & b20.05 & b30.1 & b40.2 & b5 0.4 & $\begin{array}{c}\text { Mean } \\
\text { A }\end{array}$ & b10.0 & b20.05 & b30.1 & b40.2 & b50.4 & $\begin{array}{c}\text { Mean } \\
\text { A }\end{array}$ & b10.0 & b20.05 & b30.1 & b4 0.2 & b5 0.4 & Mean A & b10.0 & b20.05 & b3 0.1 & b4 0.2 & b5 0.4 & $\begin{array}{c}\text { Mean } \\
\text { A }\end{array}$ \\
\hline $\begin{array}{c}\text { a1 62eyes } \\
(5 \times 10+6 \times 2)\end{array}$ & 22.0 & 23.0 & 24.0 & 25.0 & 25.3 & 23.9 & 23.0 & 24.1 & 25.2 & 25.3 & 25.5 & 24.6 & 91.9 & 93.0 & 94.1 & 96.0 & 96.3 & 94.3 & 92.9 & 95.0 & 96.3 & 98.0 & 98.0 & 96.0 \\
\hline $\begin{array}{l}\text { a2 72eyes } \\
(6 \times 10+6 \times 2)\end{array}$ & 22.0 & 22.0 & 23.0 & 24.0 & 24.3 & 22.7 & 22.0 & 23.3 & 25.0 & 26.6 & 26.7 & 24.7 & 93.0 & 94.9 & 95.9 & 97.0 & 97.3 & 95.6 & 94.1 & 95.1 & 96.1 & 97.9 & 100.0 & 96.6 \\
\hline $\begin{array}{l}\text { a3 82eyes } \\
(7 \times 10+6 \times 2)\end{array}$ & 18.0 & 19.0 & 20.0 & 21.0 & 22.0 & 20.0 & 20.0 & 21.8 & 23.0 & 24.1 & 24.2 & 22.6 & 94.9 & 96.0 & 97.5 & 99.0 & 99.3 & 97.3 & 95.9 & 98.1 & 99.9 & 100.0 & 100.5 & 98.9 \\
\hline $\begin{array}{l}\text { a4 92eyes } \\
(8 \times 10+6 \times 2)\end{array}$ & 16.0 & 17.1 & 18.2 & 19.3 & 19.4 & 18.0 & 19.0 & 20.6 & 21.8 & 23.0 & 24.0 & 21.7 & 96.1 & 97.1 & 98.2 & 99.1 & 99.3 & 98.0 & 97.0 & 100.0 & 102.0 & 103.0 & 103.0 & 101.0 \\
\hline $\begin{array}{l}\text { a5 102eyes } \\
(8 \times 10+6 \times 2)\end{array}$ & 14.0 & 15.5 & 16.6 & 17.7 & 17.8 & 16.3 & 18.0 & 19.1 & 20.3 & 22.0 & 22.3 & 20.3 & 97.3 & 98.3 & 100.0 & 101.9 & 102.0 & 100.0 & 99.0 & 103.0 & 105.0 & 107.0 & 107.5 & 104.3 \\
\hline Mean (B) & 18.0 & 19.3 & 20.4 & 21.4 & 21.8 & & 20.4 & 21.8 & 23.1 & 24.2 & 24.5 & & 94.5 & 95.9 & 97.1 & 98.6 & 99.8 & & 95.8 & 98.2 & 99.9 & 101.2 & 101.8 & \\
\hline New L.S.D at 5\% & \multicolumn{6}{|c|}{$\begin{array}{llc}\text { A } & \text { B } & \text { AB } \\
0.8 & \text { NS } & \text { NS } \\
\end{array}$} & \multirow{2}{*}{\multicolumn{6}{|c|}{$\begin{array}{|ccc|}\text { A } & \text { B } & \text { AB } \\
0.8 & \text { NS } & \text { NS } \\
\end{array}$}} & \multicolumn{6}{|c|}{$\begin{array}{lll}\text { A } & \text { B } & \text { AB } \\
\text { 0.9 } & \text { NS } & \text { NS }\end{array}$} & \multirow{2}{*}{\multicolumn{5}{|c|}{$\begin{array}{|ccc|}\text { A } & \text { B } & \text { AB } \\
1.0 & \text { NS } & \text { NS } \\
\text { veight/vine (kg) } & & \\
\end{array}$}} & \\
\hline \multirow{3}{*}{ Character } & \multicolumn{6}{|c|}{$\begin{array}{l}\text { Wood ripening coefficient } \\
\end{array}$} & & & & & & & \multicolumn{7}{|c|}{ Pruning wood weight/vine (kg) } & & & & & \\
\hline & \multicolumn{6}{|c|}{2014} & \multicolumn{6}{|c|}{2015} & \multicolumn{6}{|c|}{2014} & \multicolumn{6}{|c|}{\begin{tabular}{|r|}
2015 \\
\end{tabular}} \\
\hline & b10.0 & b20.05 & b3 0.1 & b4 0.2 & b5 0.4 & $\begin{array}{c}\text { Mean } \\
\text { A }\end{array}$ & b10.0 & b20.05 & b30.1 & b4 0.2 & b5 0.4 & $\begin{array}{c}\text { Mean } \\
\text { A }\end{array}$ & b10.0 & b20.05 & b3 0.1 & b4 0.2 & b5 0.4 & Mean A & b10.0 & b20.05 & b3 0.1 & b4 0.2 & b5 0.4 & $\begin{array}{c}\text { Mean } \\
\text { A }\end{array}$ \\
\hline $\begin{array}{l}\text { a1 62eyes } \\
(5 \times 10+6 \times 2)\end{array}$ & 0.71 & 0.74 & 0.76 & 0.79 & 0.80 & 0.76 & 0.72 & 0.74 & 0.77 & 0.79 & 0.80 & 0.76 & 1.41 & 1.61 & 1.81 & 2.01 & 2.03 & 1.77 & 1.37 & 1.57 & 1.77 & 1.97 & 1.98 & 1.73 \\
\hline $\begin{array}{c}\text { a2 } 72 \text { eyes } \\
(6 \times 10+6 \times 2)\end{array}$ & 0.69 & 0.71 & 0.74 & 0.76 & 0.77 & 0.73 & 0.70 & 0.72 & 0.75 & 0.76 & 0.76 & 0.74 & 1.62 & 1.82 & 2.01 & 2.21 & 2.22 & 1.98 & 1.51 & 1.73 & 1.84 & 1.94 & 1.95 & 1.79 \\
\hline $\begin{array}{l}\text { a3 82eyes } \\
(7 \times 10+6 \times 2)\end{array}$ & 0.67 & 0.69 & 0.71 & 0.73 & 0.74 & 0.71 & 0.68 & 0.70 & 0.72 & 0.74 & 0.75 & 0.72 & 1.82 & 2.02 & 2.22 & 2.42 & 2.43 & 2.18 & 1.66 & 1.81 & 1.93 & 2.05 & 2.06 & 1.90 \\
\hline $\begin{array}{l}\text { a4 92eyes } \\
(8 \times 10+6 \times 2)\end{array}$ & 0.65 & 0.67 & 0.70 & 0.72 & 0.72 & 0.69 & 0.66 & 0.68 & 0.70 & 0.72 & 0.72 & 0.70 & 1.99 & 2.19 & 2.40 & 2.60 & 2.61 & 2.36 & 1.85 & 1.99 & 2.13 & 2.28 & 2.29 & 2.11 \\
\hline $\begin{array}{l}\text { a5 102eyes } \\
(8 \times 10+6 \times 2)\end{array}$ & 0.63 & 0.64 & 0.66 & 0.68 & 0.69 & 0.66 & 0.64 & 0.66 & 0.68 & 0.70 & 0.71 & 0.68 & 2.19 & 2.41 & 2.61 & 2.81 & 2.82 & 2.57 & 1.99 & 2.11 & 2.25 & 2.31 & 2.38 & 2.21 \\
\hline Mean (B) & 0.67 & 0.69 & 0.71 & 0.74 & 0.74 & & 0.68 & 0.70 & 0.72 & \begin{tabular}{|l|}
0.74 \\
\end{tabular} & 0.75 & & 1.81 & 2.01 & 2.21 & 2.41 & 2.42 & & 1.68 & 1.84 & 1.94 & 2.11 & 2.13 & \\
\hline New L.S.D at 5\% & & & 0.2 & $\begin{array}{ll}\text { B } & A \\
0.2 & 1\end{array}$ & $\begin{array}{l}\text { AB } \\
0.4\end{array}$ & & & $\begin{array}{l}\mathbf{A} \\
\mathbf{0}\end{array}$ & .2 & $\begin{array}{l}\text { B } \\
0.2\end{array}$ & $\begin{array}{c}\mathrm{AB} \\
0.4\end{array}$ & & & $\begin{array}{r}\mathrm{A} \\
\mathbf{0 . 1}\end{array}$ & & B 13 & $\begin{array}{c}\mathrm{AB} \\
0.29\end{array}$ & & & $\begin{array}{r}A \\
0.1\end{array}$ & $\begin{array}{ll}\mathbf{A} & \mathrm{I} \\
11 & 0\end{array}$ & B 12 & $\begin{array}{l}\mathrm{AB} \\
0.27\end{array}$ & \\
\hline
\end{tabular}


Table 4. Effect of different vine loads and citric acid concentrations on cane thickness and leaf pigments of Superior grapevines during 2014 and 2015 seasons.

\begin{tabular}{|c|c|c|c|c|c|c|c|c|c|c|c|c|c|c|c|c|c|c|c|c|c|c|c|c|}
\hline \multirow{4}{*}{ Vine laod levels (A) } & \multicolumn{12}{|c|}{ Cane thickness (cm) } & \multicolumn{12}{|c|}{ Chlorophyll a (mg/100g F.W) } \\
\hline & \multicolumn{6}{|c|}{2014} & \multicolumn{6}{|c|}{2015} & \multicolumn{6}{|c|}{2014} & \multicolumn{6}{|c|}{2015} \\
\hline & \multicolumn{24}{|c|}{ Citric acid concentrations (B) } \\
\hline & $\begin{array}{l}\text { b1 } \\
0.0\end{array}$ & $\begin{array}{c}\text { b2 } \\
0.05 \\
\end{array}$ & b3 0.1 & b4 0.2 & b5 0.4 & Mean A & $\begin{array}{l}\text { b1 } \\
0.0\end{array}$ & $\begin{array}{c}\text { b2 } \\
0.05 \\
\end{array}$ & $\begin{array}{l}\text { b3 } \\
0.1\end{array}$ & b4 0.2 & b5 0.4 & Mean A & \begin{tabular}{|l|} 
b1 \\
o.0 \\
\end{tabular} & $\begin{array}{c}\text { b2 } \\
0.05 \\
\end{array}$ & b3 0.1 & b4 0.2 & b5 0.4 & Mean A & \begin{tabular}{|l|}
$\mathbf{b 1}$ \\
$\mathbf{0 . 0}$ \\
\end{tabular} & $\begin{array}{c}\text { b2 } \\
0.05 \\
\end{array}$ & b3 0.1 & b4 0.2 & b5 0.4 & Mean A \\
\hline a1 62eyes $(5 \times 10+6 \times 2)$ & 0.94 & 0.99 & 1.03 & 1.08 & 1.09 & 1.02 & 0.99 & 1.08 & 1.14 & 1.19 & 1.20 & 1.12 & 4.0 & 4.5 & 4.9 & 5.4 & 5.5 & 4.9 & 3.7 & 4.4 & 5.0 & 5.5 & 5.6 & 4.8 \\
\hline a2 72eyes $(6 \times 10+6 \times 2)$ & 0.98 & 1.03 & 1.09 & 1.13 & 1.14 & 1.07 & 1.05 & 1.13 & 1.18 & 1.22 & 1.23 & 1.16 & 4.4 & 4.9 & 5.5 & 5.9 & 6.0 & 5.3 & 4.1 & 4.6 & 5.2 & 6.0 & 6.1 & 4.0 \\
\hline a3 82eyes $(7 \times 10+6 \times 2)$ & 1.02 & 1.09 & 1.14 & 1.19 & 1.20 & 1.13 & 1.10 & 1.18 & 1.23 & 1.28 & 1.29 & 1.22 & 4.8 & 5.5 & 6.0 & 6.5 & 6.6 & 5.9 & 4.6 & 5.2 & 6.0 & 6.5 & 6.6 & 5.8 \\
\hline a4 92eyes $(8 \times 10+6 \times 2)$ & 1.07 & 1.14 & 1.21 & 1.25 & 1.26 & 1.19 & 1.15 & 1.20 & 1.25 & 1.31 & 1.32 & 1.25 & 5.3 & 6.0 & 6.6 & 7.0 & 7.1 & 6.4 & 5.1 & 6.0 & 6.4 & 7.0 & 7.1 & 6.3 \\
\hline a5 102eyes $(8 \times 10+6 \times 2)$ & 1.11 & 1.22 & 1.27 & 1.32 & 1.33 & 1.25 & 1.19 & 1.25 & 1.31 & 1.35 & 1.36 & 1.29 & 5.7 & 6.6 & 7.0 & 7.5 & 7.6 & 6.9 & 5.6 & 6.4 & 7.1 & 7.6 & 7.7 & 6.9 \\
\hline Mean (B) & 1.02 & 1.09 & 1.15 & 1.19 & 1.20 & & 1.10 & 1.17 & 1.22 & 1.27 & 1.28 & & 4.8 & 5.5 & 6.0 & 6.5 & 6.6 & & 4.6 & 5.3 & 5.9 & 6.5 & 6.6 & \\
\hline New L.S.D at 5\% & & & $\begin{array}{l}\text { A } \\
0.8 \\
\end{array}$ & $\begin{array}{ll}\text { B } & \text { A } \\
\text { NS } & 1 \\
\end{array}$ & & & & & $\begin{array}{l}\mathrm{A} \\
0\end{array}$ & $\begin{array}{|ll|}8 & \text { B } \\
\end{array}$ & B & & & & $\begin{array}{l}\text { A } \\
0.9 \\
\end{array}$ & $\begin{array}{ll}\mathrm{B} & \mathrm{Al} \\
\mathrm{NS} & \mathrm{N} \\
\end{array}$ & & & & & $\begin{array}{l}\text { A } \\
1.0 \\
\end{array}$ & $\begin{array}{ll}\text { B } & \text { AB } \\
\text { NS } & \text { NS } \\
\end{array}$ & & \\
\hline \multirow{3}{*}{ Character } & \multicolumn{12}{|c|}{ Chlorophyll b (mg/100g F.W) } & \multicolumn{12}{|c|}{ Total chlorophylls (mg/100g F.W) } \\
\hline & \multicolumn{6}{|c|}{2014} & \multicolumn{6}{|c|}{2015} & \multicolumn{6}{|c|}{2014} & \multicolumn{6}{|c|}{2015} \\
\hline & $\begin{array}{l}\text { b1 } \\
0.0 \\
\end{array}$ & $\begin{array}{c}\text { b2 } \\
0.05 \\
\end{array}$ & b3 0.1 & b4 0.2 & b5 0.4 & Mean A & $\begin{array}{l}\text { b1 } \\
0.0\end{array}$ & $\begin{array}{c}\text { b2 } \\
0.05 \\
\end{array}$ & $\begin{array}{l}\text { b3 } \\
0.1 \\
\end{array}$ & b4 0.2 & b5 0.4 & Mean A & $\begin{array}{l}\text { b1 } \\
0.0 \\
\end{array}$ & $\begin{array}{c}\text { b2 } \\
\mathbf{0 . 0 5} \\
\end{array}$ & b3 0.1 & b4 0.2 & b5 0.4 & Mean A & \begin{tabular}{|l|} 
b1 \\
0.0 \\
\end{tabular} & $\begin{array}{c}\text { b2 } \\
\mathbf{0 . 0 5} \\
\end{array}$ & b3 0.1 & b4 0.2 & b5 0.4 & Mean A \\
\hline a1 62eyes $(5 \times 10+6 x 2)$ & 1.2 & 1.6 & 1.9 & 2.2 & 2.0 & 1.8 & 1.4 & 1.6 & 2.0 & 2.3 & 2.4 & 1.9 & 5.2 & 6.1 & 6.8 & 7.6 & 7.5 & 6.6 & 5.1 & 6.0 & 7.0 & 7.8 & 8.0 & 6.8 \\
\hline a2 72eyes $(6 \times 10+6 \times 2)$ & 1.5 & 1.9 & 2.2 & 2.5 & 2.5 & 2.1 & 1.6 & 2.0 & 2.3 & 2.6 & 2.7 & 2.2 & 5.9 & 6.8 & 7.7 & 8.4 & 8.5 & 7.5 & 5.7 & 6.6 & 7.5 & 8.6 & 8.8 & 7.4 \\
\hline a3 82eyes $(7 \times 10+6 \times 2)$ & 1.8 & 2.2 & 2.5 & 2.8 & 2.9 & 2.4 & 1.9 & 2.3 & 2.7 & 2.9 & 3.0 & 2.6 & 6.6 & 7.7 & 8.5 & 9.3 & 9.5 & 8.3 & 6.5 & 7.5 & 8.7 & 9.4 & 9.6 & 8.3 \\
\hline a4 92eyes $(8 \times 10+6 \times 2)$ & 2.0 & 2.5 & 2.9 & 3.2 & 3.3 & 2.8 & 2.2 & 2.7 & 2.9 & 3.3 & 3.3 & 2.9 & 7.3 & 8.5 & 9.5 & 10.2 & 10.4 & 9.2 & 7.3 & 8.7 & 9.3 & 10.3 & 10.4 & 9.2 \\
\hline a5 102eyes $(8 \times 10+6 \times 2)$ & 2.2 & 2.9 & 3.2 & 3.5 & 3.6 & 3.1 & 2.5 & 2.9 & 3.3 & 3.6 & 3.7 & 3.2 & 7.9 & 9.5 & 10.2 & 11.0 & 11.2 & 10.0 & 8.1 & 9.3 & 10.4 & 11.2 & 11.4 & 10.1 \\
\hline Mean (B) & 1.7 & 2.2 & 2.5 & 2.8 & 2.9 & & 1.9 & 2.3 & 2.6 & 2.9 & 3.0 & & 6.6 & 7.7 & 8.5 & 9.3 & 9.4 & & 6.5 & 7.6 & 8.6 & 9.5 & 9.6 & \\
\hline New L.S.D at 5\% & \multicolumn{6}{|c|}{$\begin{array}{lll}\text { A } & \text { B } & \text { AB } \\
0.2 & 0.2 & 0.4\end{array}$} & \multicolumn{6}{|c|}{$\begin{array}{lc}\text { B } & \text { AB } \\
0.2 & 0.4\end{array}$} & \multicolumn{6}{|c|}{$\begin{array}{llc}\text { A } & \text { B } & \text { AB } \\
0.4 & 0.4 & 0.9\end{array}$} & \multicolumn{6}{|c|}{$\begin{array}{lll}\text { A } & \text { B } & \text { AB } \\
0.4 & 0.4 & 0.9\end{array}$} \\
\hline
\end{tabular}


Table 5. Effect of different vine loads and citric acid concentrations on the percentage of $\mathrm{N}, \mathrm{P}, \mathrm{K}$ and $\mathrm{Mg}$ in the leaves of Superior grapevines during 2014 and 2015 seasons.

\begin{tabular}{|c|c|c|c|c|c|c|c|c|c|c|c|c|c|c|c|c|c|c|c|c|c|c|c|c|}
\hline \multirow{4}{*}{ Vine load levels (A) } & \multicolumn{12}{|c|}{ Leaf $N \%$} & \multicolumn{12}{|c|}{ Leaf P \% } \\
\hline & \multicolumn{6}{|c|}{2014} & \multicolumn{6}{|c|}{2015} & \multicolumn{6}{|c|}{2014} & \multicolumn{6}{|c|}{2015} \\
\hline & \multicolumn{24}{|c|}{ Citric acid concentrations (B) } \\
\hline & $\begin{array}{l}\text { b1 } \\
0.0\end{array}$ & $\begin{array}{c}\text { b2 } \\
\mathbf{0 . 0 5}\end{array}$ & $\begin{array}{l}\text { b3 } \\
0.1 \\
\end{array}$ & $\begin{array}{l}\text { b4 } \\
0.2\end{array}$ & $\begin{array}{l}\text { b5 } \\
0.4\end{array}$ & $\begin{array}{c}\text { Mean } \\
\text { A }\end{array}$ & \begin{tabular}{l|} 
b1 \\
0.0
\end{tabular} & \begin{tabular}{|c|} 
b2 \\
0.05 \\
\end{tabular} & $\begin{array}{l}\text { b3 } \\
0.1\end{array}$ & $\begin{array}{l}\text { b4 } \\
0.2\end{array}$ & $\begin{array}{l}b 5 \\
0.4\end{array}$ & $\begin{array}{c}\text { Mean } \\
\text { A }\end{array}$ & \begin{tabular}{l|} 
b1 \\
0.0
\end{tabular} & \begin{tabular}{c|} 
b2 \\
0.05
\end{tabular} & $\begin{array}{l}\text { b3 } \\
0.1 \\
\end{array}$ & $\begin{array}{l}\text { b4 } \\
0.2\end{array}$ & $\begin{array}{l}\text { b5 } \\
0.4\end{array}$ & $\begin{array}{c}\text { Mean } \\
\text { A }\end{array}$ & $\begin{array}{l}\text { b1 } \\
0.0\end{array}$ & \begin{tabular}{|c|} 
b2 \\
0.05 \\
\end{tabular} & $\begin{array}{l}\text { b3 } \\
0.1\end{array}$ & $\begin{array}{l}\text { b4 } \\
0.2\end{array}$ & $\begin{array}{l}\text { b5 } \\
0.4\end{array}$ & $\begin{array}{c}\text { Mean } \\
\text { A }\end{array}$ \\
\hline a1 62eyes $(5 \times 10+6 \times 2)$ & 1.60 & 1.66 & 1.71 & 1.79 & 1.80 & 1.71 & 1.66 & 1.73 & 1.81 & 1.90 & 1.91 & 1.80 & 0.17 & 0.19 & 0.21 & 0.23 & 0.24 & 0.21 & 0.18 & 0.20 & 0.22 & 0.24 & 0.25 & 0.22 \\
\hline a2 72 eyes $(6 \times 10+6 \times 2)$ & 1.66 & 1.71 & 1.79 & 1.86 & 1.87 & 1.78 & 1.72 & 1.81 & 1.90 & 1.97 & 1.98 & 1.88 & 0.20 & 0.23 & 0.26 & 0.28 & 0.29 & 0.25 & 0.21 & 0.22 & 0.25 & 0.27 & 0.28 & 0.25 \\
\hline a3 82eyes $(7 \times 10+6 \times 2)$ & 1.73 & 1.80 & 1.89 & 1.95 & 1.96 & 1.87 & 1.81 & 1.91 & 2.00 & 2.11 & 2.12 & 1.99 & 0.22 & 0.25 & 0.28 & 0.30 & 0.31 & 0.27 & 0.24 & 0.27 & 0.30 & 0.32 & 0.33 & 0.29 \\
\hline a4 92eyes $(8 \times 10+6 \times 2)$ & 1.80 & 1.86 & 1.92 & 2.00 & 2.01 & 1.92 & 1.90 & 1.99 & 2.11 & 2.20 & 2.21 & 2.08 & 0.25 & 0.28 & 0.31 & 0.33 & 0.33 & 0.30 & 0.25 & 0.28 & 0.31 & 0.33 & 0.33 & 0.30 \\
\hline $\begin{array}{ll}5 & 102 \text { eyes } \\
(8 \times 10+6 \times 2) & \\
\end{array}$ & 1.81 & 1.92 & 2.00 & 2.11 & 2.12 & 1.99 & 1.99 & 2.11 & 2.20 & 2.30 & 2.31 & 2.18 & 0.28 & 0.30 & 0.33 & 0.35 & 0.36 & 0.32 & 0.27 & 0.30 & 0.33 & 0.35 & 0.35 & 0.32 \\
\hline Mean (B) & 1.72 & 1.79 & 1.86 & 1.94 & 1.95 & & 1.82 & 1.91 & 2.00 & 2.10 & 2.11 & & 0.22 & 0.25 & 0.28 & 0.30 & 0.31 & & 0.23 & 0.25 & 0.28 & 0.30 & 0.31 & \\
\hline New L.S.D at 5\% & & & $\begin{array}{c}\mathrm{A} \\
0.05\end{array}$ & $\begin{array}{c}\text { B } \\
0.05 \\
\end{array}$ & $\begin{array}{l}\mathrm{AB} \\
0.11\end{array}$ & & & & $\begin{array}{l}\text { A } \\
0.05\end{array}$ & $\begin{array}{c}\text { B } \\
0.05\end{array}$ & $\begin{array}{l}\mathrm{AB} \\
0.11\end{array}$ & & & & $\begin{array}{c}\mathrm{A} \\
0.02\end{array}$ & $\begin{array}{c}\text { B } \\
0.02 \\
\end{array}$ & $\begin{array}{l}\text { AB } \\
0.05\end{array}$ & & & & $\begin{array}{c}\mathrm{A} \\
\mathbf{0 . 0 2} \\
\end{array}$ & $\begin{array}{c}\text { B } \\
0.02 \\
\end{array}$ & $\begin{array}{l}\text { AB } \\
0.05\end{array}$ & \\
\hline \multirow{3}{*}{ Character } & \multicolumn{12}{|c|}{ Leaf K \% } & \multicolumn{12}{|c|}{ Leaf $\mathrm{Mg} \%$} \\
\hline & \multicolumn{6}{|c|}{2014} & \multicolumn{6}{|c|}{2015} & \multicolumn{6}{|c|}{2014} & \multicolumn{6}{|c|}{2015} \\
\hline & $\begin{array}{l}\text { b1 } \\
0.0 \\
\end{array}$ & $\begin{array}{c}\text { b2 } \\
0.05\end{array}$ & $\begin{array}{l}\text { b3 } \\
0.1 \\
\end{array}$ & $\begin{array}{ll}\text { b4 } \\
0.2 \\
\end{array}$ & $\begin{array}{l}\text { b5 } \\
0.4 \\
\end{array}$ & $\begin{array}{c}\text { Mean } \\
\text { A }\end{array}$ & $\begin{array}{l}\text { b1 } \\
0.0 \\
\end{array}$ & \begin{tabular}{|c|} 
b2 \\
0.05 \\
\end{tabular} & $\begin{array}{l}\text { b3 } \\
0.1 \\
\end{array}$ & $\begin{array}{l}\text { b4 } \\
0.2 \\
\end{array}$ & $\begin{array}{l}\text { b5 } \\
0.4 \\
\end{array}$ & $\begin{array}{c}\text { Mean } \\
\text { A }\end{array}$ & \begin{tabular}{|l|l} 
b1 \\
0.0 \\
\end{tabular} & $\begin{array}{c}\text { b2 } \\
0.05 \\
\end{array}$ & $\begin{array}{l}\text { b3 } \\
0.1 \\
\end{array}$ & \begin{tabular}{|c|} 
b4 \\
0.2 \\
\end{tabular} & $\begin{array}{l}\text { b5 } \\
0.4 \\
\end{array}$ & $\begin{array}{c}\text { Mean } \\
\text { A }\end{array}$ & $\begin{array}{l}\text { b1 } \\
0.0 \\
\end{array}$ & \begin{tabular}{|c|} 
b2 \\
0.05 \\
\end{tabular} & $\begin{array}{l}\text { b3 } \\
0.1 \\
\end{array}$ & \begin{tabular}{|l|} 
b4 \\
0.2 \\
\end{tabular} & $\begin{array}{l}\text { b5 } \\
0.4 \\
\end{array}$ & $\begin{array}{c}\text { Mean } \\
\text { A }\end{array}$ \\
\hline a1 62eyes $(5 \times 10+6 \times 2)$ & 1.41 & 1.51 & 1.60 & 1.66 & 1.67 & 1.57 & 1.36 & 1.41 & 1.47 & 1.55 & 1.56 & 1.47 & 0.50 & 0.55 & 0.60 & 0.66 & 0.67 & 0.60 & 0.50 & 0.55 & 0.58 & 0.61 & 0.62 & 0.57 \\
\hline a2 72 eyes $(6 \times 10+6 \times 2)$ & 1.48 & 1.60 & 1.66 & 1.75 & 1.76 & 1.65 & 1.41 & \begin{tabular}{|l|}
1.47 \\
\end{tabular} & 1.55 & 1.61 & 1.62 & 1.53 & 0.54 & 0.61 & 0.67 & 0.71 & 0.72 & 0.65 & 0.55 & \begin{tabular}{|l|l}
0.59 \\
\end{tabular} & 0.62 & 0.66 & 0.67 & 0.62 \\
\hline a3 82eyes $(7 \times 10+6 \times 2)$ & 1.56 & 1.62 & 1.68 & 1.74 & 1.75 & 1.67 & 1.47 & 1.55 & 1.61 & 1.69 & 1.70 & 1.66 & 0.57 & 0.64 & 0.64 & 0.74 & 0.75 & 0.67 & 0.59 & 0.64 & 0.70 & 0.76 & 0.77 & 0.69 \\
\hline a4 92eyes $(8 \times 10+6 \times 2)$ & 1.64 & 1.69 & 1.76 & 1.84 & 1.85 & 1.76 & 1.59 & \begin{tabular}{|l|l|}
1.60 \\
\end{tabular} & 1.66 & 1.71 & 1.72 & 1.66 & 0.61 & 0.70 & 0.76 & 0.81 & 0.82 & 0.74 & 0.63 & 0.68 & 0.72 & 0.76 & 0.77 & 0.71 \\
\hline $\begin{array}{ll}\mathbf{a 5} & 102 \mathrm{eyes} \\
(8 \times 10+6 \times 2) & \\
\end{array}$ & 1.71 & 1.80 & 1.86 & 1.92 & 1.93 & 1.84 & 1.64 & 1.71 & 1.76 & 1.84 & 1.85 & 1.76 & 0.65 & 0.75 & 0.80 & 0.84 & 0.85 & 0.78 & 0.67 & 0.71 & 0.77 & 0.82 & 0.83 & 0.76 \\
\hline Mean (B) & 1.56 & 1.64 & 1.71 & 1.78 & 1.79 & & 1.49 & 1.55 & 1.61 & 1.68 & 1.69 & & 0.57 & 0.65 & 0.69 & 0.75 & 0.76 & & 0.59 & 0.63 & 0.68 & 0.72 & 0.73 & \\
\hline New L.S.D at 5\% & & & $\begin{array}{c}\mathrm{A} \\
0.05\end{array}$ & $\begin{array}{c}\text { B } \\
0.05 \\
\end{array}$ & $\begin{array}{l}\mathrm{AB} \\
0.11\end{array}$ & & & & $\begin{array}{l}\text { A } \\
0.04\end{array}$ & $\begin{array}{c}\text { B } \\
0.04\end{array}$ & $\begin{array}{l}\mathrm{AB} \\
0.09\end{array}$ & & & & $\begin{array}{c}\mathrm{A} \\
\mathbf{0 . 0 2} \\
\end{array}$ & $\begin{array}{c}\text { B } \\
0.02 \\
\end{array}$ & $\begin{array}{l}\text { AB } \\
0.05\end{array}$ & & & & $\begin{array}{c}\mathrm{A} \\
\mathbf{0 . 0 2}\end{array}$ & $\begin{array}{cc}\text { B } \\
0.02 \\
\end{array}$ & $\begin{array}{l}\text { AB } \\
0.05\end{array}$ & \\
\hline
\end{tabular}


Table 6. Effect of different vine loads and citric acid concentrations on bud behaviour of Superior grapevines during 2014 and 2015 seasons.

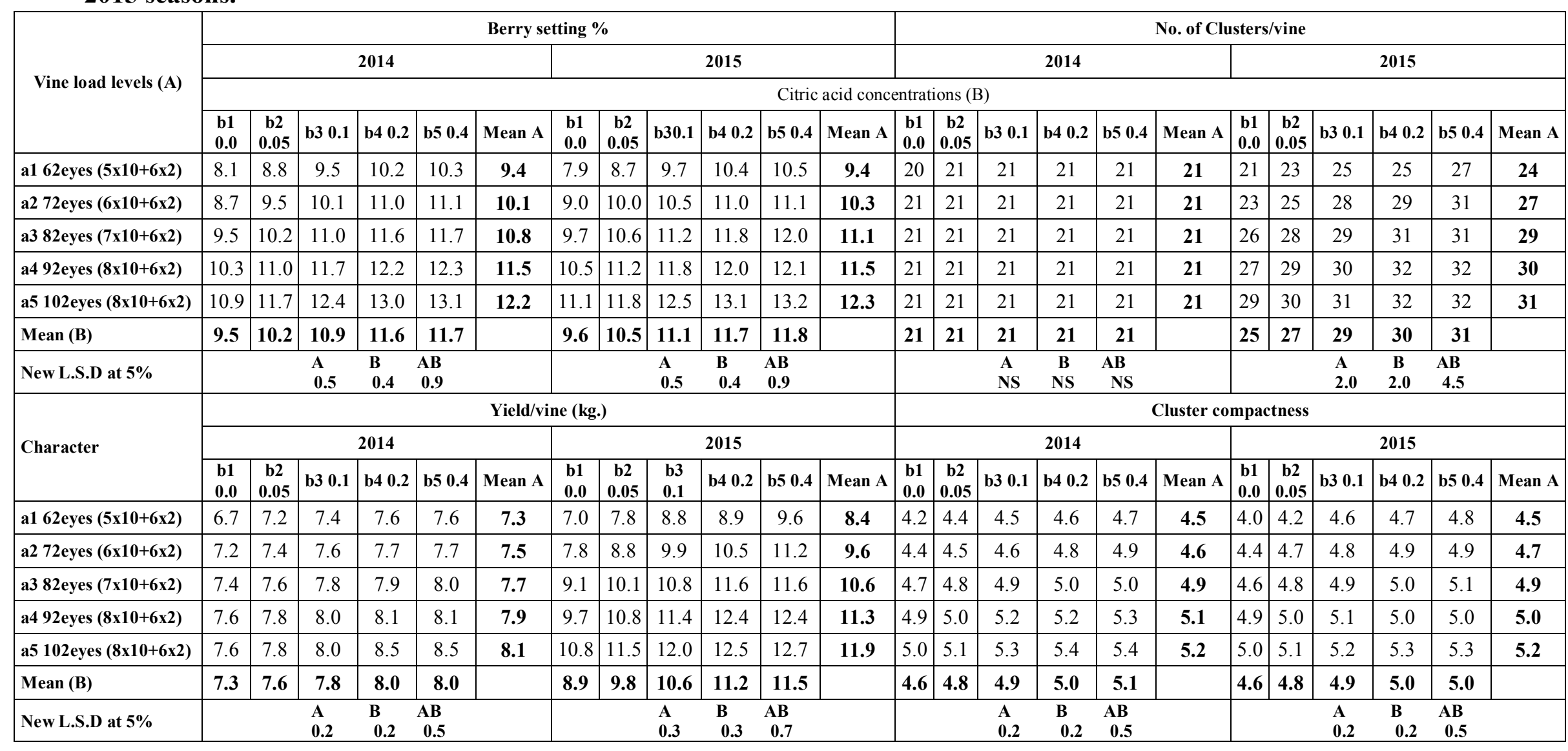


Table 7. Effect of different vine loads and citric acid concentrations on weight, length and shoulder of cluster and percentage of shot berries of Superior grapevines during 2014 and 2015 seasons.

\begin{tabular}{|c|c|c|c|c|c|c|c|c|c|c|c|c|c|c|c|c|c|c|c|c|c|c|c|c|}
\hline \multirow{4}{*}{ Vine load levels (A) } & \multicolumn{12}{|c|}{ Cluster weight (g) } & \multicolumn{12}{|c|}{ Cluster length (cm) } \\
\hline & \multicolumn{6}{|c|}{2014} & \multicolumn{6}{|c|}{2015} & \multicolumn{6}{|c|}{2014} & \multicolumn{6}{|c|}{2015} \\
\hline & \multicolumn{24}{|c|}{ Citric acid concentrations (B) } \\
\hline & \begin{tabular}{|l|}
$b 1$ \\
0.0
\end{tabular} & $\begin{array}{c}\text { b2 } \\
0.05\end{array}$ & b3 0.1 & b4 0.2 & b5 0.4 & Mean A & \begin{tabular}{|l|} 
b1 \\
0.0
\end{tabular} & \begin{tabular}{|c|}
$\mathbf{b 2}$ \\
0.05
\end{tabular} & \begin{tabular}{|l|l|} 
b3 & \\
0.1 & \\
\end{tabular} & b4 0.2 & b5 0.4 & Mean A & \begin{tabular}{|l|} 
b1 \\
o.0
\end{tabular} & \begin{tabular}{|c|c|}
$b 2$ & $b$ \\
0.05 & $b$ \\
\end{tabular} & b3 0.1 & b4 0.2 & b5 0.4 & Mean A & \begin{tabular}{|l|l|}
$b 1$ & \\
0.0 & \\
\end{tabular} & \begin{tabular}{|c|}
$\mathbf{b 2}$ \\
$\mathbf{0 . 0 5}$
\end{tabular} & b3 0.1 & $\mid$ b4 0.2 & b5 0.4 & Mean A \\
\hline a1 62eyes $(5 \times 10+6 \times 2)$ & 333 & 343 & 353 & 363 & 364 & 351 & 331 & 341 & 351 & 355 & 356 & 347 & 19.1 & 20.0 & 20.9 & 22.0 & 22.1 & 20.8 & 19.9 & 20.5 & 21.1 & 22.0 & 22.1 & 21.1 \\
\hline a2 $72 \operatorname{eyes}(6 \times 10+6 \times 2)$ & 343 & 353 & 363 & 366 & 367 & 358 & 341 & 351 & 355 & 361 & 361 & 354 & 19.6 & 20.9 & 21.9 & 22.7 & 22.8 & 21.6 & $\left.20.5\right|^{2}-2 \cos \mid$ & 21.1 & 22.0 & 22.6 & 22.7 & 21.8 \\
\hline a3 82eyes $(7 \times 10+6 \times 2)$ & 353 & 363 & 373 & 378 & 379 & 369 & 351 & 361 & 361 & 374 & 375 & 366 & 20.3 & 21.4 & 22.5 & 23.1 & 23.2 & 22.1 & $21.0 \mid 2$ & 22.0 & 22.9 & 23.4 & 23.5 & 22.6 \\
\hline a4 92eyes $(8 \times 10+6 \times 2)$ & 363 & 373 & 383 & 385 & 386 & 378 & 361 & 371 & 371 & 386 & 387 & 377 & 21.0 & 21.9 & 22.7 & 23.3 & 23.4 & 22.5 & 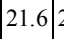 & 22.3 & 23.1 & 24.1 & 24.1 & 23.0 \\
\hline a5 102eyes $(8 \times 10+6 \times 2)$ & 375 & 385 & 390 & 394 & 395 & 388 & 372 & 382 & 382 & 391 & 376 & 386 & 21.8 & 22.8 & 23.3 & 24.0 & 24.1 & 23.2 & 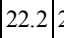 & 23.1 & 24.0 & 24.9 & 25.0 & 23.8 \\
\hline Mean (B) & 353 & 363 & 372 & 377 & 378 & & 351 & 369 & 361 & 373 & 375 & & 20.4 & 21.4 & 22.1 & 23.0 & 23.1 & & $\left.21.0\right|_{2} ^{2} \cos$ & 21.8 & 22.6 & 23.4 & 23.5 & \\
\hline New L.S.D at 5\% & & & $\begin{array}{c}\text { A } \\
10.0\end{array}$ & $\begin{array}{l}\text { B } \\
9.9\end{array}$ & $\begin{array}{l}\text { AB } \\
22.1\end{array}$ & & & & $\begin{array}{l}\text { A } \\
9.9\end{array}$ & $\begin{array}{l}\text { B } \\
9.0\end{array}$ & $\begin{array}{l}\text { AB } \\
20.2\end{array}$ & & & & $\begin{array}{l}\text { A } \\
0.4\end{array}$ & $\begin{array}{c}\text { B } \\
0.4\end{array}$ & $\begin{array}{c}\text { AB } \\
0.9\end{array}$ & & & & $\begin{array}{l}\text { A } \\
0.4\end{array}$ & $\begin{array}{c}\text { B } \\
0.4\end{array}$ & $\begin{array}{c}\text { AB } \\
0.9\end{array}$ & \\
\hline \multirow{3}{*}{ Character } & \multicolumn{12}{|c|}{ Cluster shoulder (cm) } & \multicolumn{12}{|c|}{ Shot berries \% } \\
\hline & \multicolumn{6}{|c|}{2014} & \multicolumn{6}{|c|}{2015} & \multicolumn{6}{|c|}{2014} & \multicolumn{6}{|c|}{2015} \\
\hline & $\begin{array}{c}\text { b1 } \\
0.0\end{array}$ & $\begin{array}{c}\text { b2 } \\
0.05\end{array}$ & b3 0.1 & b4 0.2 & b5 0.4 & $\mid$ Mean A & \begin{tabular}{|l|}
$b 1$ \\
0.0
\end{tabular} & \begin{tabular}{|c|}
$\mathbf{b 2}$ \\
0.05
\end{tabular} & \begin{tabular}{|l|}
$b 3$ \\
0.1
\end{tabular} & b4 0.2 l & b5 0.4 & Mean A & \begin{tabular}{l|} 
b1 \\
0.0
\end{tabular} & \begin{tabular}{|c|c|}
$b 2$ \\
0.05
\end{tabular} \mid & b3 0.1 & b4 0.2 & b5 0.4 & Mean A & $\begin{array}{l}\text { b1 } \\
0.0\end{array}$ & \begin{tabular}{|c|}
$b 2$ \\
0.05
\end{tabular} \mid & b3 0.1 & $\mid$ b4 0.2 & b5 0.4 & Mean A \\
\hline a1 62eyes $(5 \times 10+6 \times 2)$ & 9.1 & 9.7 & 10.4 & 11.0 & 11.6 & 10.4 & 8.9 & 9.5 & 10.0 & 10.5 & 10.6 & 9.9 & 7.1 & 6.0 & 5.0 & 4.0 & 3.9 & 5.2 & 7.8 & 6.9 & 6.0 & 5.0 & 4.9 & 6.1 \\
\hline a2 $72 \operatorname{eyes}(6 \times 10+6 \times 2)$ & 9.5 & 10.4 & 11.0 & 11.7 & 11.8 & 10.9 & 9.4 & 10.0 & 10.9 & 11.5 & 11.6 & 10.7 & 7.5 & 6.6 & 6.0 & 5.5 & 5.4 & 6.2 & 8.4 & 7.3 & 6.6 & 6.0 & 5.9 & 6.8 \\
\hline a3 82eyes $(7 \times 10+6 \times 2)$ & 10.0 & $|10.7|$ & 11.5 & 11.9 & 12.0 & 11.2 & 10.0 & 10.9 & 11.5 & 12.0 & 12.1 & 11.3 & 8.1 & 7.1 & 6.5 & 6.0 & 5.9 & 6.7 & 9.0 & 7.9 & 7.1 & 6.5 & 6.4 & 7.4 \\
\hline a4 92eyes $(8 \times 10+6 \times 2)$ & 10.4 & 11.5 & 12.0 & 12.5 & 12.6 & 11.8 & 10.5 & 11.3 & 12.0 & 12.7 & 12.8 & 59.3 & 8.7 & 7.9 & 7.0 & 6.6 & 6.5 & 7.3 & 10.1 & 8.4 & 7.6 & 7.0 & 6.9 & 8.0 \\
\hline a5 102eyes $(8 \times 10+6 \times 2)$ & 10.9 & 11.5 & 12.0 & 12.9 & 13.0 & 12.1 & 10.9 & 12.0 & 12.7 & 13.5 & 13.6 & 12.5 & 9.5 & 8.5 & 7.4 & 6.6 & 6.4 & 7.7 & 10.6 & 9.3 & 8.3 & 7.6 & 7.5 & 8.7 \\
\hline Mean (B) & 10.0 & 10.8 & 11.4 & 12.0 & 12.2 & & 9.9 & $|10.7|$ & 11.4 & 12.0 & 12.1 & & 8.2 & 7.2 & 6.4 & 5.7 & 5.6 & & 9.2 & 8.0 & 7.1 & 6.4 & 6.3 & \\
\hline New L.S.D at 5\% & & & $\begin{array}{l}\text { A } \\
0.3\end{array}$ & $\begin{array}{l}\text { B } \\
0.3\end{array}$ & $\begin{array}{c}\text { AB } \\
0.7\end{array}$ & & & & $\begin{array}{l}\text { A } \\
0.3\end{array}$ & $\begin{array}{l}\text { B } \\
0.3\end{array}$ & $\begin{array}{c}\text { AB } \\
0.7\end{array}$ & & & & $\begin{array}{l}\text { A } \\
0.4\end{array}$ & $\begin{array}{l}\text { B } \\
0.3\end{array}$ & $\begin{array}{c}\text { AB } \\
0.7\end{array}$ & & & & $\begin{array}{l}\text { A } \\
0.3\end{array}$ & $\begin{array}{l}\text { B } \\
0.3\end{array}$ & $\begin{array}{c}\text { AB } \\
0.7\end{array}$ & \\
\hline
\end{tabular}


Table 8. Effect of different vine loads and citric acid concentrations on some physical characteristics of the berries of Superior grapevines during 2014 and 2015 seasons.

\begin{tabular}{|c|c|c|c|c|c|c|c|c|c|c|c|c|c|c|c|c|c|c|c|c|c|c|c|c|}
\hline \multirow{4}{*}{ Vine load levels (A) } & \multicolumn{12}{|c|}{ Berry weight (g.) } & \multicolumn{12}{|c|}{ Berry longitudinal (cm) } \\
\hline & \multicolumn{6}{|c|}{2014} & \multicolumn{6}{|c|}{2015} & \multicolumn{6}{|c|}{2014} & \multicolumn{6}{|c|}{2015} \\
\hline & \multicolumn{24}{|c|}{ Citric acid concentrations (B) } \\
\hline & \begin{tabular}{|l|} 
b1 \\
0.0
\end{tabular} & $\begin{array}{c}\text { b2 } \\
0.05\end{array}$ & b3 0.1 & b4 0.2 & b5 0.4 & Mean A & \begin{tabular}{|l|} 
b1 \\
0.0
\end{tabular} & $\mid \begin{array}{c}\text { b2 } \\
0.05\end{array}$ & \begin{tabular}{|c|} 
\\
$b 3$ \\
0.1
\end{tabular} & b4 0.2 & b5 0.4 & Mean A & \begin{tabular}{l|} 
b1 \\
0.0
\end{tabular} & $\begin{array}{c}\text { b2 } \\
0.05\end{array}$ & b3 0.1 & b4 0.2 & b5 0.4 & Mean A & \begin{tabular}{|l|} 
b1 \\
0.0
\end{tabular} & \begin{tabular}{|c|} 
b2 \\
0.05
\end{tabular} & b3 0.1 & b4 0.2 & b5 0.4 & Mean A \\
\hline a1 62eyes $(5 \times 10+6 \times 2)$ & 3.91 & 3.96 & 4.00 & 4.07 & 4.08 & 4.00 & 4.00 & 4.11 & 4.20 & 4.26 & 4.27 & 4.17 & 1.99 & 2.02 & 2.05 & 2.09 & 2.10 & 2.06 & 1.98 & 2.01 & 2.03 & 2.05 & 2.06 & 2.03 \\
\hline a2 $72 \operatorname{eyes}(6 \times 10+6 \times 2)$ & 3.81 & 3.86 & 3.90 & 3.96 & 3.97 & 3.90 & 3.90 & 3.96 & 6.00 & 4.10 & 4.11 & 3.92 & 1.96 & 1.99 & 2.01 & 2.03 & 2.04 & 2.01 & 1.90 & 1.94 & 1.96 & 1.98 & 1.99 & 1.95 \\
\hline a3 82eyes $(7 \times 10+6 x 2)$ & 3.76 & 3.81 & 3.86 & 3.90 & 3.90 & 3.85 & 3.84 & 3.88 & 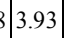 & 3.98 & 3.99 & 3.86 & 1.93 & 1.95 & 51.98 & 2.00 & 2.01 & 1.97 & 1.86 & 1.90 & 1.92 & 1.94 & 1.95 & 1.91 \\
\hline a4 92eyes $(8 \times 10+6 \times 2)$ & 3.70 & 3.76 & 3.81 & 3.81 & 3.88 & 3.80 & 3.78 & 3.82 & 3.87 & 3.91 & 3.92 & 3.82 & 1.90 & 1.92 & 1.94 & 1.96 & 1.97 & 1.94 & 1.81 & 1.87 & 1.89 & 1.90 & 1.94 & 1.88 \\
\hline a5 102eyes $(8 \times 10+6 \times 2)$ & 3.66 & 3.70 & 3.76 & 3.80 & 3.81 & 3.75 & 3.72 & 3.77 & 3.82 & 3.88 & 3.89 & & 1.87 & 1.89 & 1.91 & 1.93 & 1.93 & 1.91 & 1.77 & 1.79 & 1.80 & 1.83 & 1.84 & 1.81 \\
\hline Mean (B) & 3.77 & 3.82 & 3.87 & 3.92 & 3.93 & & 3.85 & 3.91 & 13.96 & 4.03 & 4.04 & & 1.93 & 1.95 & \begin{tabular}{|l|l}
5 & 1.99 \\
\end{tabular} & 2.00 & 2.01 & & 1.86 & 1.90 & $\mid 1.92$ & 1.94 & 1.98 & \\
\hline New L.S.D at 5\% & & & $\begin{array}{c}\text { A } \\
0.05\end{array}$ & $\begin{array}{c}\text { B } \\
0.04\end{array}$ & $\begin{array}{c}\mathrm{AB} \\
0.09\end{array}$ & & & & $\begin{array}{c}\mathrm{A} \\
0.05\end{array}$ & $\begin{array}{c}\text { B } \\
0.05\end{array}$ & $\begin{array}{c}\text { AB } \\
0.11\end{array}$ & & & & $\begin{array}{c}A \\
0.02\end{array}$ & $\begin{array}{c}\text { B } \\
0.02\end{array}$ & $\begin{array}{c}\text { AB } \\
0.05\end{array}$ & & & & $\begin{array}{c}A \\
0.02\end{array}$ & $\begin{array}{c}\text { B } \\
0.02\end{array}$ & $\begin{array}{c}\text { AB } \\
0.05\end{array}$ & \\
\hline \multirow{3}{*}{ Character } & \multicolumn{12}{|c|}{ Berry equatorial (cm) } & \multicolumn{12}{|c|}{ Juice \% } \\
\hline & \multicolumn{6}{|c|}{2014} & \multicolumn{6}{|c|}{2015} & \multicolumn{6}{|c|}{2014} & \multicolumn{6}{|c|}{2015} \\
\hline & \begin{tabular}{|l|} 
b1 \\
0.0
\end{tabular} & \begin{tabular}{c|} 
b2 \\
0.05
\end{tabular} & b3 0.1 & b4 0.2 & b5 0.4 & Mean A & $\begin{array}{l}\text { b1 } \\
\text { 0.0 }\end{array}$ & $\begin{array}{c}\text { b2 } \\
0.05\end{array}$ & \begin{tabular}{|c|} 
\\
53 \\
0.1
\end{tabular} & b4 0.2 & b5 0.4 & Mean A & \begin{tabular}{l|l|} 
b1 \\
0.0
\end{tabular} & \begin{tabular}{|c|}
$\mathbf{b 2}$ \\
$\mathbf{0 . 0 5}$
\end{tabular} & b3 0.1 & b4 0.2 & b5 0.4 & Mean A & $\begin{array}{l}\text { b1 } \\
0.0\end{array}$ & \begin{tabular}{c|}
$\mathbf{b 2}$ \\
0.05
\end{tabular} & b3 0.1 & b4 0.2 & b5 0.4 & Mean A \\
\hline a1 62eyes $(5 \times 10+6 \times 2)$ & 1.90 & 1.94 & 1.98 & 1.99 & 1.99 & 1.96 & 1.89 & 1.91 & 1.94 & 1.95 & 1.95 & 1.93 & 75.5 & 76.0 & 76.3 & 77.7 & 77.9 & 76.8 & 76.1 & 76.9 & 77.9 & 78.0 & 78.1 & 77.4 \\
\hline a2 $72 \operatorname{eyes}(6 \times 10+6 \times 2)$ & 1.86 & 1.88 & 1.91 & 1.93 & 1.94 & 1.90 & 1.86 & 1.88 & 1.91 & 1.93 & 1.94 & 1.90 & 74.9 & 75.5 & 76.6 & 77.0 & 77.1 & 76.2 & 75.3 & 76.0 & 76.9 & 77.3 & 77.3 & 76.6 \\
\hline a3 82eyes $(7 \times 10+6 x 2)$ & 1.82 & 1.84 & 1.86 & 1.88 & 1.90 & 1.86 & 1.84 & 1.87 & \begin{tabular}{l|l|l|}
7 & 1.90 \\
\end{tabular} & 1.92 & 1.93 & 1.89 & 74.0 & 74.9 & 75.6 & 76.1 & 76.2 & 75.4 & 74.8 & 75.9 & 76.3 & 76.8 & 76.9 & 76.1 \\
\hline a4 92eyes $(8 \times 10+6 \times 2)$ & 1.80 & 1.82 & 1.85 & 1.86 & 1.87 & 1.84 & 1.81 & 1.83 & \begin{tabular}{|l|l|} 
& 1.85 \\
\end{tabular} & 1.87 & 1.88 & 1.85 & 73.5 & 74.0 & 74.6 & 75.3 & 75.4 & 74.6 & 74.0 & 74.9 & 75.5 & 75.9 & 76.0 & 75.3 \\
\hline a5 102eyes $(8 \times 10+6 \times 2)$ & 1.78 & 1.80 & 1.81 & 1.83 & 1.84 & 1.81 & 1.79 & 1.81 & 1.82 & 1.84 & 1.85 & 1.82 & 73.0 & 73.5 & 74.0 & 74.8 & 75.0 & 74.1 & 73.1 & 74.7 & 75.0 & 75.6 & 75.8 & 75.0 \\
\hline Mean (B) & 1.83 & 1.86 & 1.88 & 1.90 & 1.91 & & 1.84 & 1.86 & $6 \mid 1.88$ & 1.90 & 1.91 & & 74.2 & 74.8 & 75.5 & 76.2 & 76.3 & & 74.8 & 75.7 & 76.3 & 76.7 & 76.8 & \\
\hline New L.S.D at 5\% & & & $\begin{array}{c}\mathrm{A} \\
0.02\end{array}$ & $\begin{array}{c}\text { B } \\
0.02\end{array}$ & $\begin{array}{c}\mathrm{AB} \\
0.05\end{array}$ & & & & $\begin{array}{c}\mathrm{A} \\
0.02\end{array}$ & $\begin{array}{c}\text { B } \\
0.02\end{array}$ & $\begin{array}{l}\text { AB } \\
0.05\end{array}$ & & & & $\begin{array}{l}\text { A } \\
0.4\end{array}$ & $\begin{array}{l}\text { B } \\
0.4\end{array}$ & $\begin{array}{l}\text { AB } \\
0.9\end{array}$ & & & & $\begin{array}{l}\mathrm{A} \\
0.3\end{array}$ & $\begin{array}{l}\text { B } \\
0.4\end{array}$ & $\begin{array}{c}\text { AB } \\
0.9\end{array}$ & \\
\hline
\end{tabular}


Table 9. Effect of different vine loads and citric acid concentrations on some chemical characteristics of the berries of Superior grapevines during 2014 and 2015 seasons.

\begin{tabular}{|c|c|c|c|c|c|c|c|c|c|c|c|c|c|c|c|c|c|c|c|c|c|c|c|c|}
\hline \multirow{4}{*}{$\begin{array}{l}\text { Vine load } \\
\text { levels (A) }\end{array}$} & \multicolumn{12}{|c|}{ T.S.S\% } & \multicolumn{12}{|c|}{ Titratable acidity \% } \\
\hline & \multicolumn{6}{|c|}{2014} & \multicolumn{6}{|c|}{2015} & \multicolumn{6}{|c|}{2014} & \multicolumn{6}{|c|}{2015} \\
\hline & \multicolumn{24}{|c|}{ Citric acid concentrations (B) } \\
\hline & \begin{tabular}{l|} 
b1 \\
0.0
\end{tabular} & \begin{tabular}{|c|}
$b 2$ \\
0.05 \\
\end{tabular} & b3 0.1 & b4 0.2 & b5 0.4 & Mean A & $\begin{array}{l}\text { b1 } \\
0.0\end{array}$ & \begin{tabular}{|c|}
$b 2$ \\
0.05 \\
\end{tabular} & $\begin{array}{l}\text { b3 } \\
0.1\end{array}$ & b4 0.2 & $\begin{array}{ll}2 & b 50\end{array}$ & Mean A & \begin{tabular}{|l|l|} 
b1 & 1 \\
0.0 & 0 \\
\end{tabular} & \begin{tabular}{c|c} 
b2 & \\
0.05 & b
\end{tabular} & b3 0.1 & b4 0.2 & b5 0.4 & Mean A & \begin{tabular}{|l|l} 
b1 & \\
0.0 & 0 \\
\end{tabular} & \begin{tabular}{|c|} 
b2 \\
0.05
\end{tabular} & b3 0.1 & b4 0.2 & b5 0.4 & Mean A \\
\hline $\begin{array}{l}\text { a1 } 62 \text { eyes } \\
(5 \times 10+6 \times 2)\end{array}$ & 17.5 & 17.8 & 18.1 & 18.5 & 18.5 & 18.1 & 18.0 & 18.4 & 18.8 & 19.2 & 19.2 & 18.70 & 0.6990. & 0.6800 & 0.660 & 0.640 & 0.639 & $\mathbf{0 . 6 6 4} 0$ & 0.7190 & 0.7010 & 0.681 & 0.661 & 0.640 & 0.680 \\
\hline $\begin{array}{l}\text { a2 } 72 \text { eyes } \\
(6 \times 10+6 \times 2)\end{array}$ & 17.9 & 18.2 & 18.5 & 18.7 & 18.8 & 18.4 & 18.4 & 18.7 & 19.0 & 19.2 & 19. & 18.9 & 0.6800. & 0.6600 & 0.640 & 0.620 & 0.619 & 0.6440 & 0.7010 & $0.681 \mid 0$ & 0.662 & 0.640 & 0.620 & 0.661 \\
\hline $\begin{array}{l}\text { a3 82eyes } \\
(7 \times 10+6 \times 2)\end{array}$ & 18.1 & 18.4 & $\mid 18.6$ & 18.9 & 19.0 & 18.6 & 18.8 & 19.1 & 19.5 & 19.9 & 20.1 & 19.5 & 0.6610. & 0.6410 & 0.620 & 0.600 & 0.600 & 0.6240 & 0.6800 & 0.660 & 0.641 & 0.621 & 0.601 & 0.641 \\
\hline $\begin{array}{l}\text { a4 92eyes } \\
(8 \times 10+6 \times 2)\end{array}$ & 18.4 & 18.7 & 19.0 & 19.3 & 19.4 & 19.0 & 19.2 & 19.6 & 20.0 & 20.5 & 20. & 20.0 & 0.6400. & 0.6190 & 0.600 & 0.599 & 0.599 & $\mathbf{0 . 6 1 1} 0$ & 0.6600 & 0.6400 & 0.620 & 0.600 & 0.580 & 0.620 \\
\hline $\begin{array}{l}\text { a5 102eyes } \\
(8 \times 10+6 \times 2)\end{array}$ & 18.8 & 19.1 & 19.5 & 19.9 & 19.9 & 19.4 & 19.6 & 20.1 & 20.5 & 20.9 & $21 .($ & 20.4 & 0.6180. & 0.5990 & 0.571 & 0.551 & 0.520 & $\mathbf{0 . 5 7 2} 0$ & 0.6400 & 0.6200 & 0.600 & 0.580 & 0.580 & 0.604 \\
\hline Mean (B) & 18.1 & 18.4 & 18.7 & 19.1 & 19.1 & & 18.8 & 19.2 & 19.6 & 19.9 & 20.0 & & \begin{tabular}{ll|l}
0.660 & 0. \\
\end{tabular} & $0.640 \mathrm{C}$ & 0.618 & 0.602 & 0.595 & & 0.6800 & 0.680 & \begin{tabular}{|l|l|}
0.641 \\
\end{tabular} & 0.620 & 0.604 & \\
\hline $\begin{array}{l}\text { New L.S.D at } \\
5 \%\end{array}$ & & & $\begin{array}{l}\text { A } \\
0.3\end{array}$ & $\begin{array}{l}\text { B } \\
0.3\end{array}$ & $\begin{array}{l}\text { AB } \\
0.7\end{array}$ & & & & $\begin{array}{l}\text { A } \\
0.3\end{array}$ & $\begin{array}{l}\text { B } \\
0.3\end{array}$ & $\begin{array}{l}\mathbf{A} \\
\mathbf{0}\end{array}$ & & & $\begin{array}{c}\mathrm{A} \\
0.017\end{array}$ & & $\begin{array}{l}\text { B } \\
0.016\end{array}$ & $\begin{array}{r}\mathrm{AB} \\
0.03\end{array}$ & & & $\begin{array}{c}\mathrm{A} \\
0.015\end{array}$ & & $\begin{array}{l}\text { B } \\
0.015\end{array}$ & $\begin{array}{r}\mathrm{AB} \\
0.03\end{array}$ & 34 \\
\hline \multirow{3}{*}{ Character } & \multicolumn{12}{|c|}{ T.S.S./acid } & \multicolumn{12}{|c|}{ Reduce sugars \% } \\
\hline & \multicolumn{6}{|c|}{2014} & \multicolumn{6}{|c|}{2015} & \multicolumn{6}{|c|}{2014} & \multicolumn{6}{|c|}{2015} \\
\hline & $\begin{array}{l}\text { b1 } \\
0.0\end{array}$ & \begin{tabular}{c|}
$b 2$ \\
0.05
\end{tabular} & b3 0.1 & b4 0.2 & b5 0.4 & Mean A & \begin{tabular}{|l} 
b1 \\
0.0
\end{tabular} \mid & $\begin{array}{c}\mathbf{b 2} \\
\mathbf{0 . 0 5}\end{array}$ & $\begin{array}{l}b 3 \\
0.1\end{array}$ & b4 0.2 & $\begin{array}{lll}2 & \text { b5 } 0 .\end{array}$ & Mean A & \begin{tabular}{|l|l} 
b1 & b \\
0.0 & o
\end{tabular} & \begin{tabular}{c|c}
$b 2$ & $b$ \\
0.05 & $b$
\end{tabular} & b3 0.1 & b4 0.2 & $\left|\begin{array}{lll}\text { b5 } & 0.4\end{array}\right|$ & Mean A & \begin{tabular}{|l|l} 
b1 & \\
0.0 & 0 \\
\end{tabular} & \begin{tabular}{|c|c}
$b 2$ & $b$ \\
0.05 & $b$
\end{tabular} & b3 0.1 & b4 0.2 & b5 0.4 & Mean A \\
\hline $\begin{array}{l}\text { a1 } 62 \text { eyes } \\
(5 \times 10+6 \times 2)\end{array}$ & 25.0 & 26.2 & 27.4 & 28.9 & 29.0 & 27.3 & 25.0 & 26.2 & 27.6 & 29.0 & 30.0 & 27.6 & \begin{tabular}{l|l}
15.1 & 1.
\end{tabular} & 15.4 & 15.9 & 16.3 & 16.4 & 15.8 & \begin{tabular}{l|l}
15.2 & 1
\end{tabular} & 15.6 & 16.0 & 16.3 & 16.4 & 16.0 \\
\hline $\begin{array}{l}\text { a2 } 72 \text { eyes } \\
(6 \times 10+6 \times 2)\end{array}$ & 26.3 & 27.6 & 28.9 & 30.2 & 30.4 & 27.7 & 26.2 & 27.5 & 28.4 & 30.0 & 31.1 & 28.6 & \begin{tabular}{|l|l}
15.5 & 1 \\
\end{tabular} & 15.9 & 16.4 & 17.0 & 17.1 & 16.4 & \begin{tabular}{l|l}
15.9 & 1
\end{tabular} & 16.4 & 16.9 & 17.3 & 17.4 & 16.8 \\
\hline $\begin{array}{l}\text { a3 } \quad \text { 82eyes } \\
(7 \times 10+6 \times 2)\end{array}$ & 27.4 & 28.7 & 30.0 & 31.5 & 31.7 & 29.9 & 27.6 & 28.9 & 30.4 & 32.0 & 33.3 & 30.4 & \begin{tabular}{|l|l|}
15.9 & 1 \\
\end{tabular} & 16.3 & 17.0 & 17.5 & 17.6 & 16.9 & \begin{tabular}{l|l}
16.4 & 1
\end{tabular} & 16.9 & 17.4 & 17.8 & 17.9 & 17.3 \\
\hline $\begin{array}{l}\text { a4 92eyes } \\
(8 \times 10+6 \times 2)\end{array}$ & 28.7 & 30.2 & 31.7 & 32.2 & 32.4 & 31.0 & 29.1 & 30.6 & 32.3 & 34.2 & 35.5 & 32.3 & \begin{tabular}{l|l}
16.4 & 1
\end{tabular} & 16.9 & 17.4 & 18.0 & 18.1 & 17.4 & \begin{tabular}{l|l}
16.9 & 1
\end{tabular} & 17.4 & 17.8 & 18.1 & 18.1 & 17.7 \\
\hline $\begin{array}{l}\text { a5 102eyes } \\
(8 \times 10+6 \times 2)\end{array}$ & 30.4 & 31.9 & 34.2 & 36.1 & 38.3 & 34.2 & 30.6 & 32.4 & 34.2 & 36.0 & 36.2 & 33.9 & \begin{tabular}{l|l}
16.8 & 1
\end{tabular} & 17.2 & 17.6 & 18.0 & 18.1 & 17.5 & \begin{tabular}{l|l}
17.5 & 1
\end{tabular} & 18.0 & 18.4 & 18.8 & 18.9 & 18.3 \\
\hline
\end{tabular}




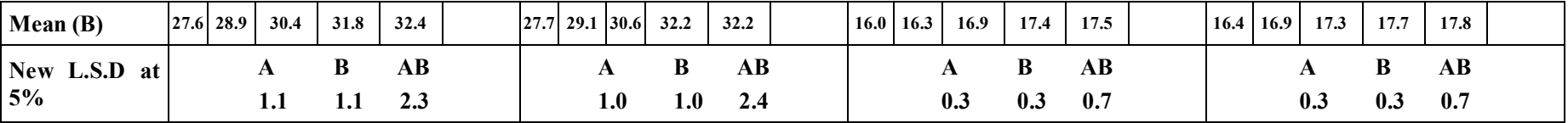




\section{References}

Abdalla, R.D. (2003): Studies on breaking dormancy of grapevines M.Sc. Thesis Fac. of Agric. Al-Azhar. Univ. Egypt.

Abdelaal, A.H.M. and Aly, M.M. (2013): The synergistic effects of using turmeric with some antioxidants on growth, vine nutritional status and productivity of Ruby seedless grapevine. Hort. Science J. of Suez Canal Univ. 1: 305-308.

Abd El- Galil, M.A.E. (2015): Response of Superior grapevines to spraying some antioxidant. M.Sc. Thesis Fac. of Agric. Minia Univ. Egypt.

Abdel-Mohsen, M.A. (2013): Application of various pruning treatments for improving productivity and fruit quality of crimson seedless grapevine. World Journal of Agricultural Sciences 9 (5): 377-382.

Ahmed, F. F and Morsy, M. H. (1999): A new method for measuring leaf area in different fruit species. Minia J. of Agric. Rec. \& Dev.19: $97-105$.

Association of Official Agricultural Chemists (2000): Official Methods of Analysis $14^{\text {th }}$ ed. A.O.A.C., Benjamin Franklin Station Washington D.C.U.S.A., pp. 490-510.

Balo, E.; Prilezky, G.; Happ, I. Kaholomi, M. and Vega, L. (1988): Soil improvement and the use of leaf analysis for forecasting nutrient requirements of grapes. Potash Review subject 2nd suite No.6.

Bouard, J. (1966): Recherches physiologiques sure et en particular sur 1004 ment des serments. Thesis Sci. Nat. Bardeux, France. P.34.

Chapman, K.R. (1990): Table grapes in the Tropics Areas for Research and Market Apportunities. Acta Horticulture: III International Workshop on Temperate Zone Fruits in the
Tropics and Subtropics 279: 910,96.

Ebrahiem, M.A.A. (2015): Response of Superior grapevines to spraying some antioxidants. M.Sc. Thesis Fac. of Agric. Minia Univ. Egypt.

El- Ashram, M.A. (1993): Modern Approaches of Cultivation and Production of Grapevines. Dar ElFekr, El- Arabie, pp. 275-276.

El- Sese, A.M.A. (2004): Effect of bud load and fertility on yield and fruit quality of Red Roomy grapevines. Assiut J. of Agric. Sci. Vol. 35 No. 4: 117-130.

Fawzi, M.I.F.; Shahin, M.F.M. and Kandil, E.A. (2010): Effect of bud load on bud behavior, yield, cluster characteristics and some biochemical contents of the can of Crimson seedless grapevines. Journal of American Science 6(12).

Gao, Y. and Cahoon, G.A. (1994): Cluster shading effects on fruit quality. Fruit skin color and anthyocyanin content and composition in reliance (Vitis hybrid). Vitis, 33(4): 205-209.

Hussein, M.A. (2009): Adjusting the suitable vine bud load as well as the optimum date and concentration of Dormex for advancing bud burst and improving productivity of Superior grapevines. Ph.D. Thesis Fac. of Agric. Minia Univ. Egypt.

Lane, J.H. and L. Eynon, 1965. Determination of reducing sugars by means of Fehling's solution with methylene blue as indicator A.O.AC. Washington D.C.U.S.A. pp: 100-110.

Martin, S.R. and Dunn, G.M. (2000): Effect of pruning time and Hydrogen cynamide on bud burst and sunsequent of (Vitis vinifera L.) variety Cabernet Sauvignon in cen- 
teral Victoria. Scientia. Hort. 128 (1), 17-22.

Mead, R., CurRnow, R. N. and Harted, A. M. (1993): Statistical methods in Agricultural and Experimental Biology. $2^{\text {nd }}$ Ed. Chapman \& Hall, London pp. 10-44.

Miller, D. P.; Howell, G. S.; Striegler. R. K.(1993): Reproductive and vegetative response of mature grapevines subjected to differential cropping stresses. American Journal of Enology and Viticulture, 44 (4), pp. 435-440. (C.F. Hort. Abst. 65:1134).

Mohamed-Attiat, A.M. (2016): Trials for alleviating the adverse effects of salinity on some grapevine cv. transplants. M.Sc. Thesis Dac. of Agric. Minia Univ. Egypt.

Mohamed, M.A.; El-Sayed, M.A.; Abdel-Aal, A.M.K and Ebrahiem, M.A.A. (2015): Response of Superior grapevines to spraying some antioxidants. World Rural Observations 7(4): 22-30.

Omar, A.J. and Abdel- Kawi, A. (2000): Optimal bud load for Thompson seedless grapevines. J. Agric. Sci. Mansoura Univ. 25(9): 5769-5777.

Oretili, J.J. (1987): Exogenus application of vitamins as regulators for growth and development of plants: Pflanzenrahr Bpdenk, 150: 375391.

Ranpise, S. A.; Patil, B. T.; More, T. A.; Birade, R. M.; Ghure, T. K., (2003): Effect of sub-cane pruning on fruitfulness and yield of grape cv Thomson seedless. Journal of Maharashtra Agricultural Universities. 27 3: 258259, Ember 2002.

Rizk-Alla, Mervat. S. and El-Zyat, H. (2005): Effect of cane length on bud behavior, yield, bunch characteristics, wood ripening and chemical contents of early Superior grapevines. J. Agric. Sci.
Mansoura Univ. 30(10): 6123 6138.

Robinson, F.A. (1973): Vitamins. Phytochemistry Vol. III: 195-198. Lawrence P. Miller (Ed.) Van Nostrand Rinhold comp. New York.

Samiullah, S.A.; Ansori, M.M and Frida, R.K. (1988): B- Vitamins in relation to crop productivity. India Res. Life Sc. pp.80-97.

Samra, N. R. (1998): Evaluation of table grape cultivars behaviour and training system in Egypt and California. Report for Egypt project of Agricultural Technology Utilization and transfer project (ATUT) pp. 55-58.

Silverstroni, O.; Intrieri, C.; Filipp Etti, I.; Lavezzi, A.; Riclomi, A. and Pezza, L. (1996): Performance of Tokay vines under mechanical and hand pruned simple curtain and traditional hand pruned Casarsa system. Revista de Viticulture e di Eno, 49(4) 39-52. (Hort. Abst, 675757).

Singh, D.V.; Srivostava, G.C. and Abdin, M.Z. (2001): Amelioration of negative effect of water stress in Cassia angustfolia by benzyladenine and / or ascorbic acid. Biologia Plaetrum 44(1): 141-143.

Sourial, R.A. Al-Ashkar, M.M. Ibrahim , Mahmoud, Z.E. (2016): Response of Superior seedless grapevines to a complex of some winter pruning treatments, two hydrogen Cyanamide sprays and thinning of shoots and bunches. Zagazig J. Res. Vol. 43 (3): 771-783.

Tamura, F.; Muraya, K. and Fujji, Y. (2002): Effects of short cane pruning in summer and long cane pruning in winter on growth and yield of the grapevine "Muscat" of Alexandria' in forcing culture condition. Hort. Res. (Japan), 1(4): 269274. (Hort. Abst., 73(5): 4269). 
Valachovic, A. and Zembery, A. (2002): Grapevine pruning the basis of viticulture in the annual cycle. Vinohard Bratislava Slovakia 40(1): 2-4 (Hort. Abst., 73(1): 118).

Valor, O. and Bautista, D. (1995): Grape production on spurs with various numbers of nodes in wine grape cultivars. Hort. Sci. 39 p. 158-167.

von Wettstein, D. (1957): Chlorophyllletale undder submikroskopische Formwechsel der Plastiden. Ex- perimental Cell Research, 12(3): 427-506.

Weaver, R. J. (1976): Grape Growing. A Wiley Interscience Publication John Wiley \& Davis, New York, London, Sydney, Tronto pp. 160175.

Wilde, S. A.; Corey, R. B.; Layer, J. G. and Voigt, G. K. (1985): Soils and Plant Analysis for Tree Culture. Mohan Primlani, Oxford \& IBH Publishing Co., New Delhi, India, p 1-142. 
تأثير حمولة البراعم ورش حامض الستريك علي الإثمار فى كرمات العنب السوبريور النامى

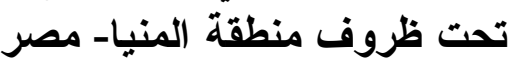

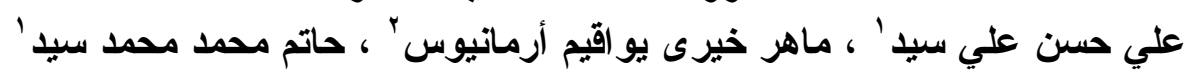

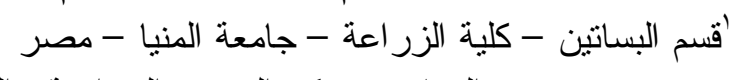

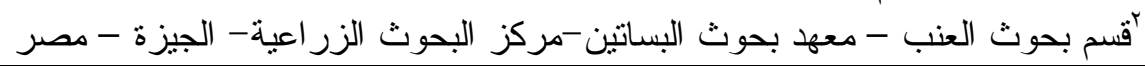

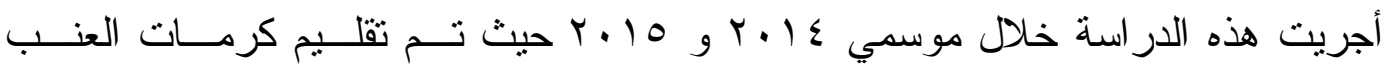

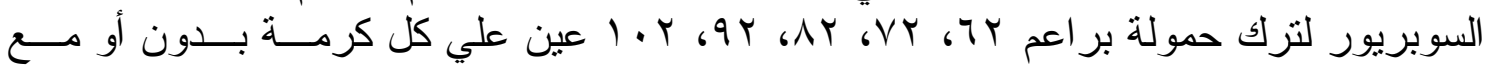

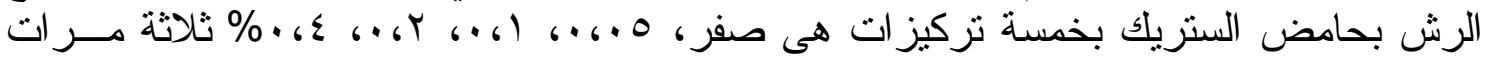

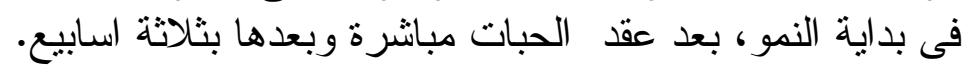

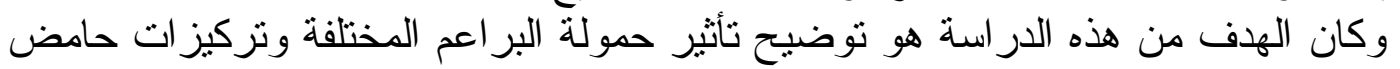

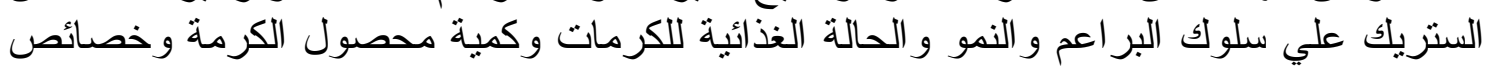

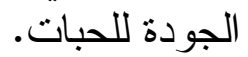

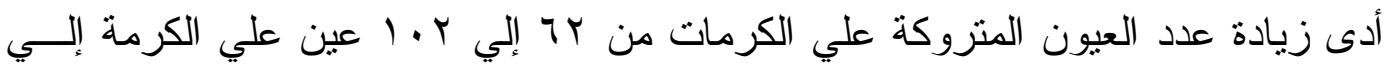

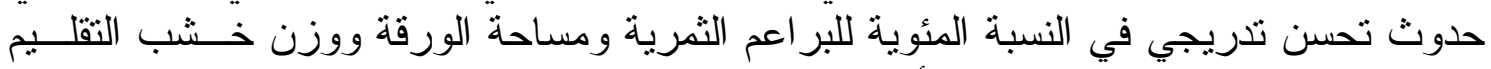

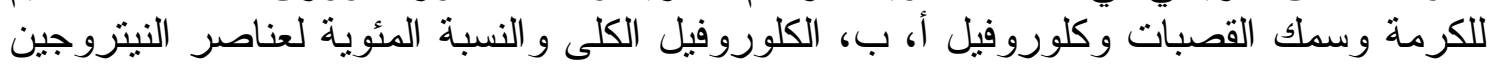

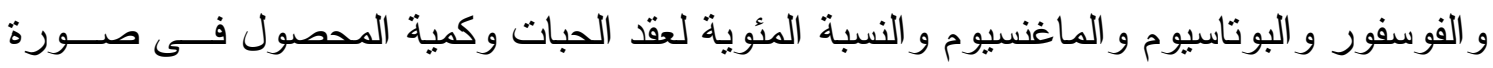

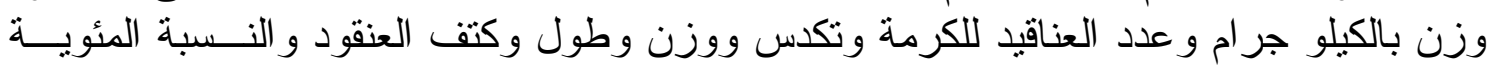

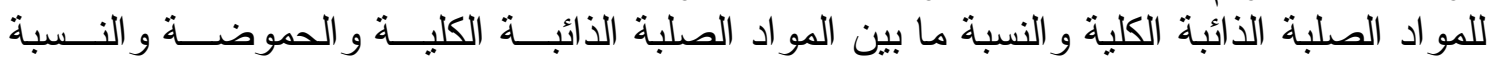

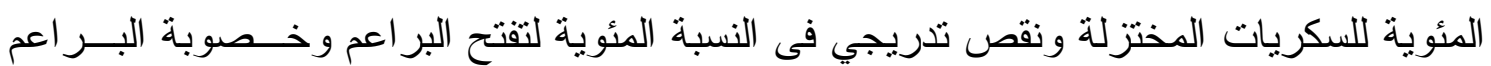

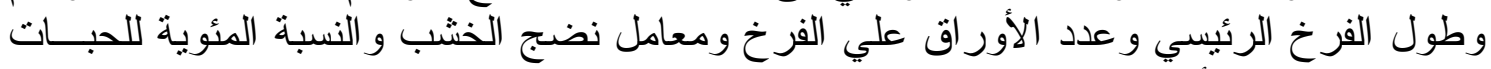

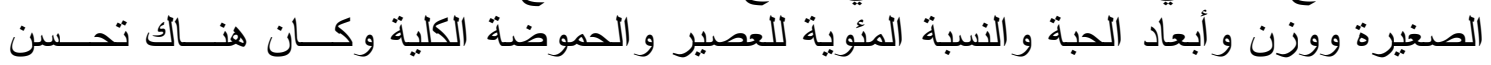

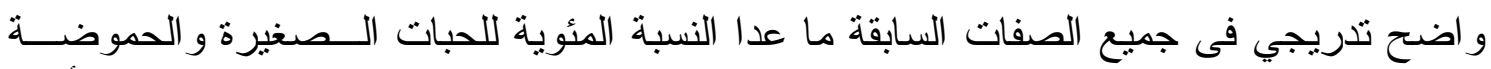

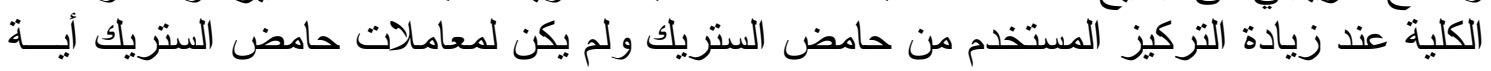

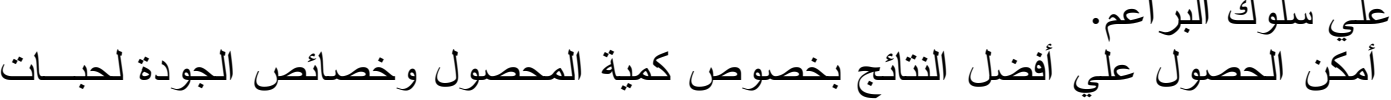

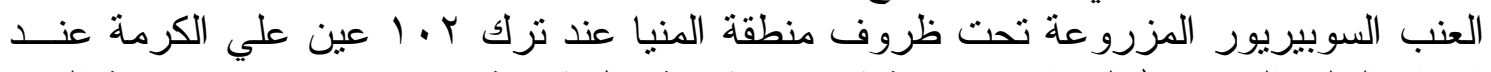

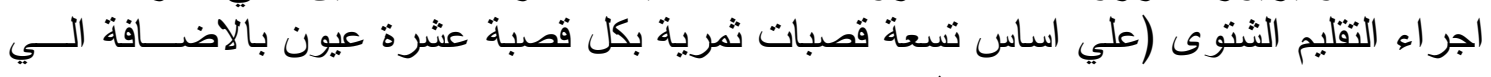

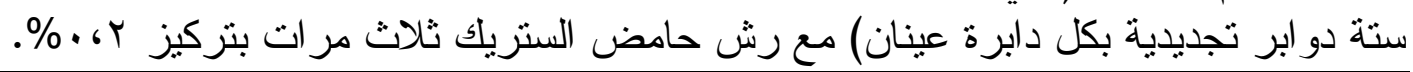

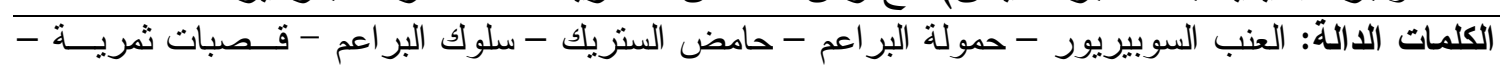
كمية المحصول - خصائص الجودة للحبات. 